\title{
A Meshless and Parallelizable Method for Differential Equations with Time-Delay
}

\author{
Shulin $\mathrm{Wu}^{1, *}$ and Chengming Huang ${ }^{2,3}$ \\ ${ }^{1}$ School of Mathematics and Statistics, Sichuan University of Science and \\ Engineering, Zigong 643000, China \\ 2 School of Mathematics and Statistics, Huazhong University of Science and \\ Technology, Wuhan 430074, China \\ ${ }^{3}$ Hubei Key Laboratory of Engineering Modeling and Scientific Computing, Huazhong \\ University of Science and Technology, Wuhan 430074, China
}

Received 7 September 2016; Accepted (in revised version) 24 March 2017

\begin{abstract}
Numerical computation plays an important role in the study of differential equations with time-delay, because a simple and explicit analytic solution is usually un available. Time-stepping methods based on discretizing the temporal derivative with some step-size $\Delta t$ are the main tools for this task. To get accurate numerical solutions, in many cases it is necessary to require $\Delta t<\tau$ and this will be a rather unwelcome restriction when $\tau$, the quantity of time-delay, is small. In this paper, we propose a method for a class of time-delay problems, which is completely meshless. The idea lies in representing the solution by its Laplace inverse transform along a carefully designed contour in the complex plane and then approximating the contour integral by the Filon-Clenshaw-Curtis (FCC) quadrature in a few fast growing subintervals. The computations of the solution for all time points of interest are naturally parallelizable and for each time point the implementations of the FCC quadrature in all subintervals are also parallelizable. For each time point and each subinterval, the FCC quadrature can be implemented by fast Fourier transform. Numerical results are given to check the efficiency of the proposed method.
\end{abstract}

AMS subject classifications: 65M15, 65D05, 65D30

Key words: Delay differential equations, meshless/parallel computatio, contour integral, FilonClenshaw-Curtis quadrature.

\section{Introduction}

Delay differential equations (DDEs) arise from various applications, like biology [27], control of dynamic systems [5,21], circuit engineering [32] and many others. A DDE differs from an ordinary differential equation (ODE) in that it depends not only on the

*Corresponding author. Email addresses: wushulin_ylp@163.com (S. L. Wu), chengming_huang@hotmail.com (C. M. Huang) 

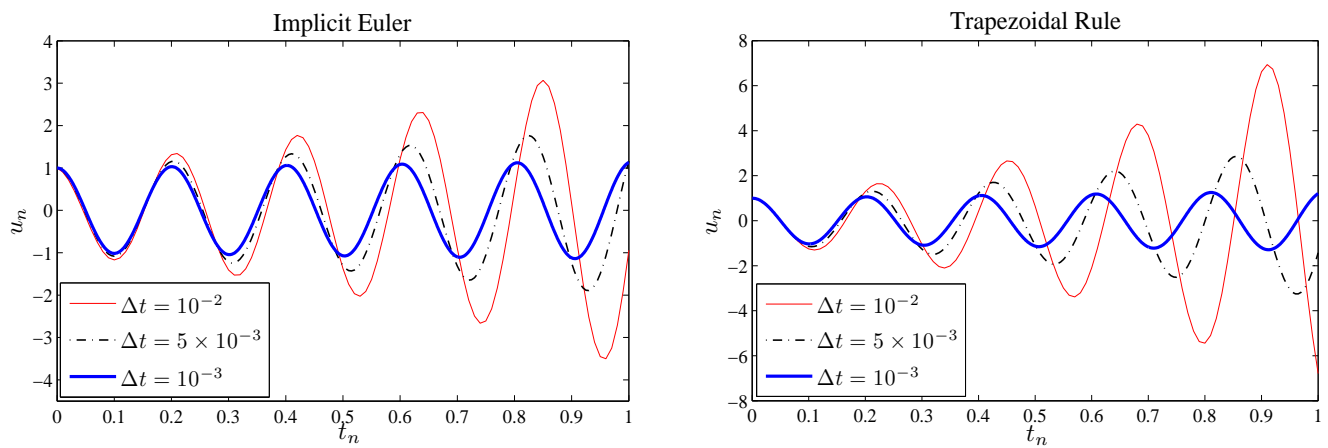

Figure 1: For DDE (1.1) with $\tau=0.05$, the approximation of the numerical solutions generated by the implicit Euler method (left) and the trapezoidal rule (right) to the exact solution, the cosine function $u(t)=\cos \left(\frac{\pi}{2 \tau} t\right)$.

solution at a present stage but also on the solution at some past stages. While the theoretical properties of DDEs have been investigated deeply and widely in the past, there is little experience with numerical methods for solving these equations, particularly with parallel computational methods. Existing numerical methods for DDEs mainly rely on discretizing the temporal derivatives with some step-size $\Delta t$ by finite difference formula and then advancing the discrete solutions step by step. For this issue, we refer the interested reader to $[3,4,13,14,34]$ and references therein. A serious limitation of the time-stepping methods is that, in many cases the step-size has to be less than the quantity of time-delay, i.e., $\Delta t<\tau$. To illustrate this, we consider the following DDE:

$$
u^{\prime}(t)+\frac{\pi}{2 \tau} u(t-\tau)=0, \quad u(t)=\cos \left(\frac{\pi}{2 \tau} t\right), \text { for } t \leq 0
$$

for which the exact solution is the cosine function $u(t)=\cos \left(\frac{\pi}{2 \tau}\right)$. Let $\tau=0.05$. Then, using the implicit Euler method and the trapezoidal rule, we get the numerical solutions for different step-sizes $\Delta t$ as shown in Fig. 1. These results imply that for DDEs a small step-size is really necessary to get accurate numerical solutions when $\tau$ is small.

Parallel methods are also studied for DDEs in the past few years. Among these, we mention $[11,17,33]$ for the study of classical waveform relaxation methods and $[22,28-30]$ for the study of the Schwarz waveform relaxation methods. (The parallelism of the former is based on system decoupling, while for the latter the parallelism is based on domain decomposition.) These methods are iterative and the convergence rates of iterations usually deteriorate as the mesh parameters become small (except a coarse grid correction is used after each iteration $[20,25,26])$. What make matters worse is that these methods can not be directly used to compute the solution of DDEs, because they are defined at the continuous level, while in practice we still need to rely on time-stepping methods to construct discrete (Schwarz) waveform relaxation methods.

The goal of this paper is to propose a meshless and highly parallelizable method for solving the following representative model problem

$$
\partial_{t} u(\mathbf{x}, t)-\Delta u(\mathbf{x}, t)+a u(\mathbf{x}, t-\tau)=f(\mathbf{x}, t), \quad(\mathbf{x}, t) \in \Omega \times(0, T),
$$


where $u(\mathbf{x}, t)=u_{0}(\mathbf{x}, t)$ for $(\mathbf{x}, t) \in \Omega \times[-\tau, 0]$ and $\Delta$ denotes the Laplacian. Our starting point is to represent the solution $u(\mathbf{x}, t)$ by its inverse Laplace transform

$$
\begin{aligned}
& u(\mathbf{x}, t)=\frac{1}{2 \pi i} \int_{\Gamma} e^{t s}\left[\left(a e^{-s \tau}+s\right) I-\Delta\right]^{-1} \hat{F}(\mathbf{x}, s) d s, \\
& \text { with } \hat{F}(\mathbf{x}, s)=\hat{f}(\mathbf{x}, s)+u_{0}(\mathbf{x}, 0)-a \int_{0}^{\tau} e^{-s r} u_{0}(\mathbf{x}, r-\tau) d r
\end{aligned}
$$

where $I$ denotes the identity operator (or identity matrix if the Laplacian $\Delta$ is approximated by a matrix $A), \hat{f}(\mathbf{x}, s)=\int_{0}^{\infty} e^{s t} f(\mathbf{x}, t) d t$ denotes the Laplace transform of the source term $f(\mathbf{x}, t)$ and $\Gamma$ denotes a suitable contour in the complex plane with opening angle to the negative real axis. Such a contour plays an important role for deriving an accurate numerical solution of (1.2) and the basic principle for contour design is that all the singularities of the integrand $\left[\left(a e^{-s \tau}+s\right) I-\Delta\right]^{-1} \hat{F}(\mathbf{x}, s)$ have to lie on the left of $\Gamma$. This issue will be addressed in detail in Section 2. The next step is to discretize the contour integral by the Filon-Clenshaw-Curtis (FCC) quadrature $[8,9,31]$. This procedure consists of three parts. First, we need to parameterize the contour $\Gamma$ as

$$
\mathbb{R} \rightarrow \Gamma: \quad y \rightarrow \theta(y), \quad-\infty<y<\infty,
$$

and rewrite the contour integral in (1.3) as

$$
\frac{1}{2 \pi i} \int_{-\infty}^{\infty} e^{t \theta(y)}\left[\left(a e^{-\theta(y) \tau}+\theta(y)\right) I-\Delta\right]^{-1} \hat{F}(\mathbf{x}, \theta(y)) \theta^{\prime}(y) d y .
$$

Second, for a given tolerance $\epsilon$ we need to truncate the infinite integral (1.5) to a finite one:

$$
\frac{1}{2 \pi i} \int_{-Y_{\max }}^{Y_{\max }} e^{t \theta(y)}\left[\left(a e^{-\theta(y) \tau}+\theta(y)\right) I-\Delta\right]^{-1} \hat{F}(\mathbf{x}, \theta(y)) \theta^{\prime}(y) d y .
$$

By using the symmetry of the integrand as a function of $y$, it suffices to compute

$$
\int_{0}^{Y_{\max }} e^{t \theta(y)}\left[\left(a e^{-\theta(y) \tau}+\theta(y)\right) I-\Delta\right]^{-1} \hat{F}(\mathbf{x}, \theta(y)) \theta^{\prime}(y) d y .
$$

The third part is to divide the interval $\left[0, Y_{\max }\right]$ into two parts $\left[0, Y_{\min }\right]$ and $\left[Y_{\min }, Y_{\max }\right]$ and further divide the second interval into a few fast growing subintervals:

$$
\begin{aligned}
{\left[Y_{\min }, Y_{\min }+B^{-J_{\min }}\right] } & \bigcup\left(\bigcup_{j=-J_{\min }}^{J_{\max }-1}\left[Y_{\min }+B^{j}, Y_{\min }+B^{j+1}\right]\right) \\
& \bigcup\left[Y_{\min }+B^{J_{\max }}, Y_{\max }\right]=\left[Y_{\min }, Y_{\max }\right],
\end{aligned}
$$


where $B^{-J_{\min }} \approx 0$ and $B^{J_{\max }} \approx Y_{\max }-Y_{\min }$. Our experience suggests that $J_{\min }=6$ and $J_{\max }=1+\left[\log _{B}\left(Y_{\max }-Y_{\min }\right)\right]$ with $B=8$ are good choice. This process reduces (1.6) to

$$
\sum_{j} \int_{Y_{j}}^{Y_{j+1}} e^{t \theta(y)}\left[\left(a e^{-\theta(y) \tau}+\theta(y)\right) I-\Delta\right]^{-1} \hat{F}(\mathbf{x}, \theta(y)) \theta^{\prime}(y) d y,
$$

with proper definitions of $\left\{Y_{j}\right\}$ (see (3.1) given in Section 3). Then, each sub-integral in (1.7) is computed by the FCC quadrature, which can be implemented in $\mathscr{O}(M \log M)$ operations by FFT (fast Fourier transform). Here, $M$ denotes the number of ClenshawCurtis points.

Clearly, the computations for all time points of interest is naturally parallelizable and we do not need any mesh to carry out the computation. For each time point, the implementations of the FCC quadrature for all the subintervals in (1.7) can be also parallelized. The major computational work is to solve a series of steady state elliptic problems of the form

$$
(z I-\Delta) U(\mathbf{x})=\hat{F}(\mathbf{x}, z), \quad \text { with } z=a e^{-\theta(y) \tau}+\theta(y) .
$$

The quantity $z$ is a complex number and satisfies $\Re(z) \rightarrow-\infty$ as $|\mathfrak{s}(z)| \rightarrow \infty$. Therefore, such a complex shift destroys the positivity of $(-\Delta)$ and this makes (1.8) a Helmholtz-like equation, which is a well known difficult problem in numerics. For this problem, Cocquet and Gander [6] make a throughout analysis for the multigrid method and the main conclusion is that: if the imaginary part of $z$ is dominate, the multigrid method possesses an ideal and robust convergence rate. This condition is fulfilled in our situation, because $|\mathfrak{I}(z)|=\mathscr{O}\left(e^{-\Re(z)}\right)$ for $\Re(z) \ll 0$. A detailed research about numerical computation of (1.8) shall be given in our forthcoming papers.

The remainder of this paper is organized as follows. In Sections 2 and 3, we supply the details for the contour integral and the FCC quadrature, respectively. Clearly, the aforementioned method depends on the Laplace forward transform of the source term $f(\mathbf{x}, t)$ and this dependence will be a problem in some situation (for example the function $\hat{f}(\mathbf{x}, s)$ is not known explicitly). In Section 4, we propose two treatments for the source term in the contour integral and for each treatment we provide numerical results for verification. We conclude this paper in Section 5.

\section{Contour design}

We start our description for the following scale model problem

$$
u^{\prime}(t)+\lambda u(t)+a u(t-\tau)=f(t), \quad u(t)=u_{0}(t), \quad \text { for } t \in[-\tau, 0],
$$

where $\lambda \geq 0$ is an arbitrary eigenvalue of $(-\Delta)$. Applying Laplace transform to (2.1) gives

$$
s \hat{u}(s)+\lambda \hat{u}(s)+a e^{-s \tau} \hat{u}(s)+a \int_{0}^{\tau} e^{-s r} u_{0}(r-\tau) d r=u_{0}(0)+\hat{f}(s),
$$


which gives $\hat{u}(s)=\left(s+a e^{-s \tau}+\lambda\right)^{-1} \hat{F}(s)$, where

$$
\hat{F}(s)=u_{0}(0)-a \int_{0}^{\tau} e^{-s r} u_{0}(r-\tau) d r+\hat{f}(s) .
$$

The methods for inverting the Laplace transform that we shall consider are based on numerical integration of the Bromwich complex contour integral

$$
u(t)=\frac{1}{2 \pi i} \int_{\sigma-i \infty}^{\sigma+i \infty} e^{s t}\left(s+a e^{-s \tau}+\lambda\right)^{-1} \hat{F}(s) d s, \quad \sigma>\sigma_{0},
$$

where $\sigma_{0}$ is a quantity such that all the singularities of $\left(s+a e^{-s \tau}+\lambda\right)^{-1} \hat{F}(s)$ lie in the open half-plane $\Re(s) \leq \sigma_{0}$. For simplicity, in this section we assume

The Laplace transform of $f$ is known and $\hat{f}(s)$ is analytic in the whole complex plane.

Then, we can focus our attention on the singularities of $\left(s+a e^{-s \tau}+\lambda\right)^{-1}$. The case that $\hat{f}(s)$ has singularities will be treated in Section 4 .

\subsection{Deform the Bromwich integral to contour integral}

The integral in (2.2) is not well-suited for numerical integration, because the exponential factor $e^{t s}$ is highly oscillatory on the Bromwich line $s=\sigma+i y(-\infty<y<\infty)$ and in general there is no guarantee that the other factor $\left(s+a e^{-s \tau}+\lambda\right)^{-1} \hat{F}(s)$ decays rapidly as $|y| \rightarrow \infty$. Integrals that are both oscillatory and slowly decaying on unbounded domains are often hard to compute. One strategy for circumventing this difficulty is based on the technique of conformal mapping, which is first used by Talbot [23] in 1979, who suggested that the Bromwich line be deformed into a contour that begins and ends in the left half-plane, such that $\Re(s) \rightarrow-\infty$ at each end of the contour. Clearly, this deformation provides decaying for the exponential factor $e^{t s}$ as $s$ moves to the ends of the contour. By Cauchy's theorem, such a deformation of contour is permissible as long as no singularities are traversed in the process. Based on this idea, we can represent $u(t)$ as

$$
u(t)=\frac{1}{2 \pi i} \int_{\Gamma} e^{s t}\left(s+a e^{-s \tau}+\lambda\right)^{-1} \hat{F}(s) d s,
$$

where $\Gamma$ is the aforementioned contour deformed from the Bromwich line and all the singularities of $\left(s+a e^{-s \tau}+\lambda\right)^{-1}$ lie on the left of $\Gamma$. Provided the decaying rate of the exponential factor $e^{t s}$ is sufficiently fast, the contour interval can be approximation by the trapezoidal rule. Three types of contours that satisfy this requirement are extensively studied in the past few years, the Talbot contour, the hyperbolic contour and the parabolic contour, which are given in the following

$$
\begin{cases}s=\theta(y)=a_{1}+a_{2} y \cot \left(a_{3} y\right)+i a_{4} y(y \in[-\pi, \pi]), & \text { Talbot, } \\ s=\theta(y)=a_{1}\left(1+\sin \left(i y-a_{2}\right)\right)(-\infty<y<\infty), & \text { Hyperbola, } \\ s=\theta(y)=\left(i y+a_{1}\right)^{2}(-\infty<y<\infty), & \text { Parabola, }\end{cases}
$$



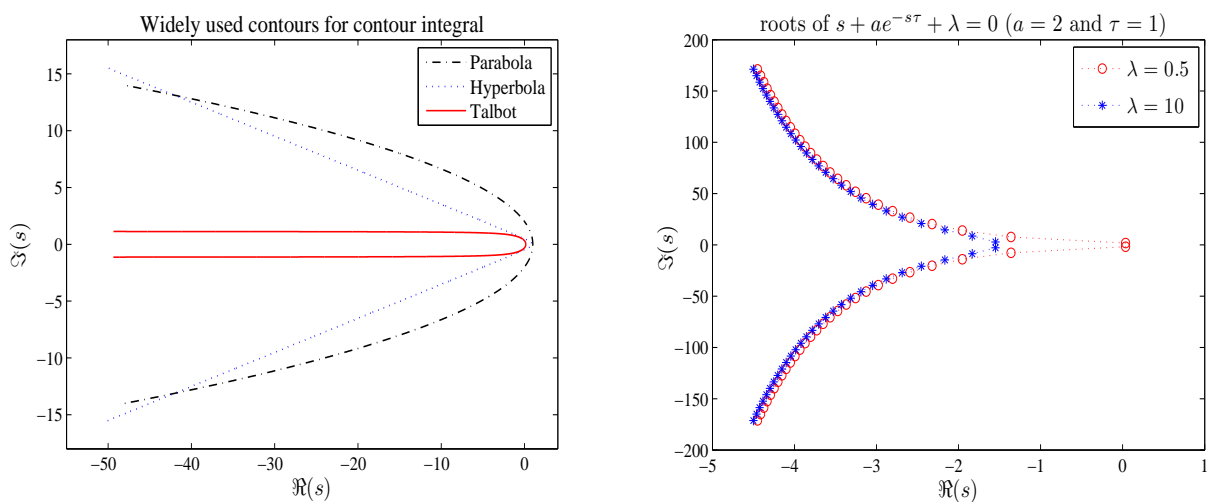

Figure 2: Left: three widely used contours in the literature for Laplace inversion. The formulas are given by (2.4) and we use the parameters $a_{1}, a_{2}, a_{3}$ and $a_{4}$ given in [24, Table 15.1]. Right: an illustration of the distribution of the roots of $s+a e^{-s \tau}+\lambda=0$ in the complex plane for two values of $\lambda$.

where $a_{1,2,3,4}$ are real constants. For more details about contour integral, we refer the interested reader to [24, Section 15]. These three contours are shown in Fig. 2 on the left.

However, none of these contours can be used in our situation! This is because, as shown in Fig. 2 on the right, the singularities of $\left(s+a e^{-s \tau}+\lambda\right)^{-1}$ satisfies $\Re(s)<0$ and $|\mathfrak{I}(s)|=\mathscr{O}(\exp (-\Re(s)))$ as $\Re(s) \rightarrow-\infty$, while for the three contours given by (2.4) it holds that

$$
\begin{cases}\frac{|\mathfrak{I}(s)|}{-\Re(s)} \rightarrow 0 \quad \text { as } \Re(s) \rightarrow-\infty, & \text { Talbot and Parabola, } \\ \frac{|\mathfrak{I}(s)|}{-\Re(s)} \rightarrow \frac{\cos \left(a_{2}\right)}{\sin \left(a_{2}\right)} \quad \text { as } \Re(s) \rightarrow-\infty, & \text { Hyperbola. }\end{cases}
$$

Lemma 2.1. For $\lambda \geq 0, \tau>0$ and $a \in \mathbb{R} \backslash\{0\}$, the complex roots of $s+a e^{-s \tau}+\lambda=0$, denoted by $s=(x, i y)$, are determined by $y=\frac{v}{\tau}$ and $x=-\frac{v \cos (v)}{\tau \sin (v)}-\lambda$, where $v$ denotes an arbitrary root of

$$
v=C^{*} E(v), \text { with } C^{*}=a \tau e^{\tau \lambda} \quad \text { and } \quad E(v)=e^{\frac{v \cos (v)}{\sin (v)}} \sin (v) .
$$

This equation has infinite number of roots and are determined as follows

1. if $C^{*}>0$ the nonlinear equation $v=C^{*} E(v)$ has a unique root $v_{k}$ in the interval $(2 k \pi,(2 k+1) \pi)$ for $k>1$ and $v_{k} \rightarrow\left(2 k \pi+\frac{\pi}{2}\right)_{-}$as $k \rightarrow \infty$;

2. if $C^{*}<0$ the nonlinear equation $v=C^{*} E(v)$ has a unique root $v_{k}$ in the interval $((2 k-1) \pi, 2 k \pi)$ for $k>1$ and $v_{k} \rightarrow\left((2 k-1) \pi+\frac{\pi}{2}\right)_{-}$as $k \rightarrow \infty$;

3. in the interval $(0, \pi), v=C^{*} E(v)$ has no root if $C^{*}<C^{\dagger}$ and a unique root if $C^{*} \geq C^{\dagger}$, where $C^{\dagger}=0.36787944118761$. 

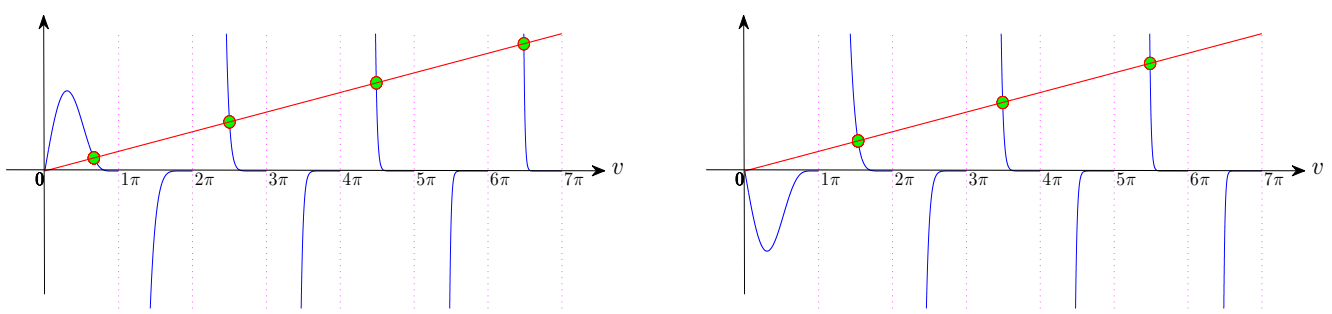

Figure 3: The intersection points between the curves $C^{*} E(v)$ (blue) and the straight line (red), which correspond to the solutions of $v=C^{*} E(v)$. Left: $C^{*}=8$; Right: $C^{*}=-8$.

Proof. Let $s=x+i y$. Then, substituting this into $s+a e^{-s \tau}+\lambda=0$ gives

$$
\left\{\begin{array}{l}
x+a e^{-\tau x} \cos (\tau y)+\lambda=0 \\
y-a e^{-\tau x} \sin (\tau y)=0
\end{array}\right.
$$

Clearly, if $(x, y)$ is a root of (2.5), $(x,-y)$ also satisfies (2.5). So, we can restrict our proof in the following to $y \geq 0$. For complex roots (i.e., $y \neq 0$ ), it mush hold $y \tau \neq k \pi$ for $k \in \mathbb{N}$. Hence, we can rewrite the second equation in (2.5) as

$$
a e^{-x \tau}=\frac{y}{\sin (\tau y)} .
$$

Inserting this into the first equation in (2.5) gives $x=-\frac{y \cos (\tau y)}{\sin (\tau y)}-\lambda$. Then, by using (2.6) we have

$$
a e^{\tau \lambda} e^{\frac{\tau y \cos (\tau y)}{\sin (\tau y)}}=\frac{y}{\sin (\tau y)} \Leftrightarrow a \tau e^{\tau \lambda} e^{\frac{\tau y \cos (\tau y)}{\sin (\tau y)}}=\frac{\tau y}{\sin (\tau y)}
$$

From this, we see that for $y \neq 0$ the roots of (2.5) are equivalent to the roots of the scalar nonlinear equation $v=C^{*} E(v)$ with $C^{*}=a \tau e^{\tau \lambda}$ and $E(v)=e^{\frac{v \cos (v)}{\sin (v)}} \sin (v)$.

A routine calculation yields

$$
E^{\prime}(v)=\frac{\sin (2 v)-v}{\sin (v)} e^{\frac{v \cos (v)}{\sin (v)}}
$$

Hence, for any integer $k \geq 1$ it holds that $E^{\prime}(v)<0$ for $v \in(2 k \pi,(2 k+1) \pi)$ and $E^{\prime}(v)>0$ for $v \in((2 k-1) \pi, 2 k \pi)$. This together with the fact that $\lim _{v \rightarrow(2 k \pi)+} E(v)=\infty$ and $\lim _{v \rightarrow((2 k+1) \pi)-} E(v)=0$ implies that $v=C^{*} E(v)$ has a unique root for $v \in[2 k \pi,(2 k+$ $1) \pi]$ if $C^{*}>0$. This equation has no root for $v \in((2 k-1) \pi, 2 k \pi)$, because in this interval $E(v) \leq 0$. For $C^{*}<0$, it is easy to know that $v=C^{*} E(v)$ has no root for $v \in$ $[2 k \pi,(2 k+1) \pi]$ and a unique root for $v \in((2 k-1) \pi, 2 k \pi)$. An illustration of the distribution of the roots of $v=C^{*} E(v)$ for $C^{*}>0$ and $C^{*}<0$ is given in Fig. 3 on the left and right, respectively.

For the first interval $(0, \pi)$, it is easy to know that $v=C^{*} E(v)$ has no root for $C^{*}<0$ since in this case $C^{*} E(v)<0$. For the case $C^{*}>0$, we denote by $\left(v^{*}, C^{*}\right)$ the solution of

$$
v=C^{*} E(v), \quad 1=C^{*} E^{\prime}(v) .
$$


Numerically, we get the solution of this nonlinear problem as $v^{\dagger}=9.37538519 \times 10^{-6}$ and $C^{\dagger}=0.36787944118761$. Then, by comparing $C^{*} E^{\prime}(v)$ with 1 , a tedious but routine calculation reveals that $v=C^{*} E(v)$ has a unique root if $C^{*} \geq C^{\dagger}$ and no root if $C^{*}<C^{\dagger}$ (see Fig. 3).

It remains to prove that the root $v_{k} \rightarrow\left(2 k \pi+\frac{\pi}{2}\right)_{-}$as $k \rightarrow \infty$, if $C^{*}>0$. Let $v_{k}=$ $2 k \pi+r_{k}$ with $r_{k} \in(0, \pi)$. It is clear that $v_{k} \rightarrow 2 k \pi+\frac{\pi}{2}$ is equivalent to $r_{k} \rightarrow\left(\frac{\pi}{2}\right)_{-}$. To prove the latter, we first prove that $r_{k} \in\left(0, \frac{\pi}{2}\right)$ as $k>\left(C^{*}-\frac{\pi}{2}\right) / 2 \pi$. This can be proven by simply noticing two facts: (1) $v-C^{*} E(v)=2 k \pi+\frac{\pi}{2}-C^{*}>0$ for $v=2 k \pi+\frac{\pi}{2}$ with $k>\left(C^{*}-\frac{\pi}{2}\right) / 2 \pi$ and (2) $E(v)$ is a decreasing function for $v \in(2 k \pi,(2 k+1) \pi)$ with $\lim _{v \rightarrow(2 k \pi)+} E(v)=\infty$ and $\lim _{v \rightarrow((2 k+1) \pi)_{-}} E(v)=0$. We now prove that the sequence $\left\{r_{k}\right\}$ converges to $\pi / 2$. We have

$$
v_{k}=C^{*} E\left(v_{k}\right) \Leftrightarrow r_{k}=C^{*} \sin \left(r_{k}\right) e^{\frac{\left(2 k \pi+r_{k}\right) \cos \left(r_{k}\right)}{\sin \left(r_{k}\right)}}-2 k \pi .
$$

For $k \gg 1$, we have $r_{k} \in\left(0, \frac{\pi}{2}\right)$ and thus $\frac{\left(2 k \pi+r_{k}\right) \cos \left(r_{k}\right)}{\sin \left(r_{k}\right)}>0$. Hence, it holds that

$$
e^{\frac{\left(2 k \pi+r_{k}\right) \cos \left(r_{k}\right)}{\sin \left(r_{k}\right)}}>\frac{\left(2 k \pi+r_{k}\right)^{2} \cos ^{2}\left(r_{k}\right)}{2 \sin ^{2}\left(r_{k}\right)}
$$

which can be derived by noticing the fact that $e^{x}>\frac{x^{2}}{2}$ for any $x>0$. Hence, for $k \gg 1$ we have

$$
\begin{aligned}
& \frac{\pi}{2}>r_{k}>C^{*} \frac{\left(2 k \pi+r_{k}\right)^{2} \cos ^{2}\left(r_{k}\right)}{2 \sin \left(r_{k}\right)}-2 k \pi \\
> & C^{*} \frac{(2 k \pi)^{2} \cos ^{2}\left(r_{k}\right)}{2}-2 k \pi, \quad \text { i.e., } \quad \cos \left(r_{k}\right)<\frac{2\left(\frac{\pi}{2}+2 k \pi\right)}{C^{*}(2 k \pi)^{2}} .
\end{aligned}
$$

Then, by letting $k \rightarrow \infty$ we have $\frac{2\left(\frac{\pi}{2}+2 k \pi\right)}{C^{*}(2 k \pi)^{2}} \rightarrow 0$, which implies $r_{k} \rightarrow\left(\frac{\pi}{2}\right)_{-}$.

If $C^{*}<0$, by a similar analysis we can also prove $v_{k} \rightarrow\left((2 k-1) \pi+\frac{\pi}{2}\right)_{-}$as $k \rightarrow \infty$.

Since the root $v_{k}$ exists uniquely in the interval $(2 k \pi,(2 k+1) \pi)$ (resp. $((2 k-1) \pi, 2 k \pi)$ for $C^{*}>0$ (resp. $C^{*}<0$ ), it can be computed conveniently by many nonlinear solvers, for example the fzero solver in Matlab. By using Lemma 2.1, we get the following asymptotic relation between the real and imaginary parts of the roots of $s+a e^{-\tau s}+\lambda=0$.

Corollary 2.1. For given $\lambda \geq 0, \tau>0$ and $a \in \mathbb{R} \backslash\{0\}$, let $\left\{s_{k}\right\}_{k \geq 1}$ be the complex roots of $s+a e^{-\tau s}+\lambda=0$ with $s_{k}=-\frac{v_{k} \cos \left(v_{k}\right)}{\tau \sin \left(v_{k}\right)}-\lambda+i \frac{v_{k}}{\tau}$ and $v_{k}$ being the unique roots of $v=C^{*} E(v)$ in the interval $(2 k \pi,(2 k+1) \pi)$ (resp. $((2 k-1) \pi, 2 k \pi))$ for $C^{*}>0$ (resp. $\left.C^{*}<0\right)$, where $C^{*}$ and $E(v)$ are given in Lemma 2.1. Then, it holds that

$$
\left|\mathfrak{s}\left(s_{k}\right)\right| \rightarrow|a| e^{-\Re\left(s_{k}\right) \tau}, \quad \text { as } k \rightarrow \infty .
$$


Proof. Let $s_{k}=x_{k}+i y_{k}$. Then, from Lemma 2.1 we have $\tau y_{k}=v_{k}$ and $v_{k} \rightarrow$ $\left(2 k \pi+\frac{\pi}{2}\right)_{-}\left(\right.$resp. $\left.v_{k} \rightarrow\left((2 k-1) \pi+\frac{\pi}{2}\right)_{-}\right)$for $C^{*}>0$ (resp. $\left.C^{*}<0\right)$ as $k \rightarrow \infty$. This implies that

$$
\sin \left(\tau y_{k}\right)=\sin \left(v_{k}\right) \rightarrow \operatorname{sign}\left(C^{*}\right)=\operatorname{sign}(a) \text { as } k \rightarrow \infty .
$$

Hence, for both $a>0$ and $a<0$ we have $\left|\sin \left(\tau y_{k}\right)\right| \rightarrow 1$ as $k \rightarrow \infty$. From (2.6), we have $a e^{-x_{k} \tau}=\frac{y_{k}}{\sin \left(\tau y_{k}\right)}$, which holds for all $k \geq 1$. This, together with $\left|\sin \left(\tau y_{k}\right)\right| \rightarrow 1$, gives $\left|y_{k}\right|=|a| e^{-x_{k} \tau}\left|\sin \left(\tau y_{k}\right)\right| \rightarrow|a| e^{-x_{k} \tau}$ as $k \rightarrow \infty$. This completes the proof of the lemma.

Lemma 2.1and Corollary 2.1 describe the distribution of the singularities of integrand in (2.3) in the complex plane, except the real axis. On the real axis, we have following result.

Lemma 2.2. Let $E_{0}(x)=x+a e^{-\tau x}+\lambda$ with $a \in \mathbb{R} \backslash\{0\}, \lambda \geq 0$ and $\tau>0$. Let $x^{*}=$ $\log (a \tau) / \tau$ for $a>0$. Then, if $a>0$ and $E_{0}\left(x^{*}\right)>0, E_{0}(x)$ has no root; otherwise

1. if a $0, E_{0}(x)$ has a unique root $x_{0} \in(-\lambda,-a)$ and $x_{0}$ decreases as $\lambda$ increases;

2. if $a>0$ and $E_{0}\left(x^{*}\right)<0, E_{0}(x)$ has two different roots $\underline{x}_{0} \in\left(-a-\lambda, x^{*}\right)$ and $x_{0} \in$ $\left(x^{*}, a\right)$ and $x_{0}$ decreases as $\lambda$ increases;

3. if $a>0$ and $E_{0}\left(x^{*}\right)=0, E_{0}(x)$ has a unique root $x_{0}=x^{*}$.

Proof. For $a<0$ it holds that $E_{0}^{\prime}(x)=1-a \tau e^{-\tau x}>0$ for all $x \in \mathbb{R}$ and $E_{0}( \pm \infty)=$ $\pm \infty$. Hence, in this case $E_{0}(x)$ has a unique root $x_{0}$. Since $E_{0}(-\lambda)=a e^{\lambda \tau}<0$ and $E_{0}(-a)=-a\left(1-e^{a \tau}\right)+\lambda>0$, it holds that $x_{0} \in(-\lambda,-a)$. This root can be regarded as the intersection point between the function $f_{1}(x):=x+a e^{-\tau x}$ and the transverse line $f_{2}(x):=-\lambda$. Since $f_{1}^{\prime}(x)=E_{0}^{\prime}(x)>0$, as $\lambda$ increases (i.e., $-\lambda$ becomes smaller), the solution of $f_{1}(x)=f_{2}(x)$ decreases.

For $a>0$ we know that $E_{0}^{\prime}(x)$ has a unique root $x=x^{*}=\log (a \tau) / \tau$ and that $E_{0}^{\prime}(x)<0$ (resp. $E_{0}^{\prime}(x)>0$ ) for $x \in\left(-\infty, x^{*}\right)$ (resp. $x \in\left(x^{*}, \infty\right)$ ). Hence, if $E_{0}\left(x^{*}\right)<0$ we have two different roots, denoted by $\underline{x}_{0}$ and $x_{0}$, which are respectively located in the intervals $\left(-\infty, x^{*}\right)$ and $\left(x^{*}, \infty\right)$. Since $E_{0}(-a-\lambda)=-a+a e^{\tau(a+\lambda)}>0$ and $E_{0}(a)>0$, we can specify a finite interval for $\underline{x}_{0}$ and $x_{0}$ as $\left(-a-\lambda, x^{*}\right)$ and $\left(x^{*}, a\right)$, respectively. The decreasing property of the larger root $x_{0}$ as $\lambda$ increases can be deduced by a similar analysis as given for the case $a<0$.

For $a>0$ and $E_{0}\left(x^{*}\right)=0$, it is clear that $E_{0}(x)$ has a unique root at $x=x^{*}$. If $a>0$ and $E_{0}\left(x^{*}\right)>0$, it always holds $E_{0}(x)>0$ and therefore $E_{0}(x)$ has no root.

Now, suppose $\lambda \geq \lambda_{\min } \geq 0$. Then, from Corollary 2.1 we know that for each $\lambda \geq \lambda_{\min }$ the singularities of $\left(s+a e^{-s \tau}+\lambda\right)^{-1}$, denoted by $\left\{s_{k}\right\}_{k \geq 1}$, approaches to the curve $|y|=$ $|a| e^{-x \tau}$ in the complex plane as $k \rightarrow \infty$. This property is independent of $\lambda$. Lemma 2.2 implies that the maximal real singularity of $\left(s+a e^{-s \tau}+\lambda\right)^{-1}$ decreases as $\lambda$ increases. These two properties motivate us to consider the following contour

$$
\Gamma_{\boldsymbol{\beta}}:=\left\{\begin{array}{l}
\left\{(x, i y):|y|=\beta_{0}+|a| e^{-\tau\left(x-\beta_{1}\right)}-\left(x-\beta_{1}\right), x<x_{0}+\beta_{0}\right\}, \\
\left\{(x, i y): y \in\left[-Y_{\min }, Y_{\min }\right], x=x_{0}+\beta_{0}\right\},
\end{array}\right.
$$


where $\boldsymbol{\beta}=\left(\beta_{0}, \beta_{1}\right)$ are free parameters,

$$
Y_{\min }=\beta_{0}+|a| e^{-\tau\left(x_{0}+\beta_{0}-\beta_{1}\right)}-\left(x_{0}+\beta_{0}-\beta_{1}\right)=\beta_{1}+|a| e^{-\tau\left(x_{0}+\beta_{0}-\beta_{1}\right)}-x_{0},
$$

and $x_{0}$ denotes the maximal real part of the roots of $s+a^{-\tau s}+\lambda_{\min }=0^{1}$. The parameters $\beta_{0}$ and $\beta_{1}$ should satisfy the following two conditions

1. $\beta_{0}>0, \beta_{1}>0$ and $Y_{\min }=\beta_{1}+|a| e^{-\tau\left(x_{0}+\beta_{0}-\beta_{1}\right)}-x_{0} \geq 0$;

2. for all $\lambda \geq \lambda_{\min }$ the singularities of $\left(s+a e^{-s \tau}+\lambda\right)^{-1}$ lie on the left of $\Gamma_{\beta}$.

We note that for any $\beta_{0}>0$ and $\beta_{1}>0$, it holds asymptotically that the singularities of ( $s+$ $\left.a e^{-s \tau}+\lambda\right)^{-1}$ lie on the left of $\Gamma_{\beta}$ for all $\lambda \geq \lambda_{\min }$. The real singularities of $\left(s+a e^{-s \tau}+\lambda\right)^{-1}$ lie on the left of $\Gamma_{\beta}$, too. However, to determine a suitable $\Gamma_{\beta}$ that satisfies the second requirement, we need to check the first few, say 15 , singularities of $\left(s+a e^{-s \tau}+\lambda_{\min }\right)^{-1}$ such that these singularities lie on the left of $\Gamma_{\beta}$. An illustration of the shape of $\Gamma_{\beta}$ (with $\boldsymbol{\beta}=(0.1,0.1))$ is shown in Fig. 4, where the singularities of $\left(s+a e^{-s \tau}+\lambda\right)^{-1}$ for three values of $\lambda$ are shown by solid lines with markers and the contour is shown by dash-dot lines.

\subsection{Parameterization and truncation of the contour integral}

We now parameterize the contour $\Gamma_{\boldsymbol{\beta}}$ given by (2.8) as a function of $y$ :

$$
s=\theta(y):= \begin{cases}X(y)+i y, & \text { for } y>Y_{\min }:=\beta_{1}+|a| e^{-\tau\left(x_{0}+\beta_{0}-\beta_{1}\right)}-x_{0}, \\ x_{0}+\beta_{0}+i y, & \text { for }-Y_{\min } \leq y \leq Y_{\min }, \\ X(y)-i y, & \text { for } y<-Y_{\min },\end{cases}
$$

where $X(y)$ is the inverse function for $x$ implied by the relation ${ }^{2}$

$$
y=\beta_{0}+|a| e^{-\tau\left(x-\beta_{1}\right)}-\left(x-\beta_{1}\right) .
$$

Then, we can represent the contour integral (2.3) as

$$
\begin{aligned}
u(t) & =\frac{1}{2 \pi i} \int_{-\infty}^{\infty} e^{\theta(y) t}\left(\theta(y)+a e^{-\theta(y) \tau}+\lambda\right)^{-1} \hat{F}(\theta(y)) \theta^{\prime}(y) d y \\
& =\frac{1}{\pi} \mathfrak{S}\left(\int_{0}^{\infty} e^{\theta(y) t}\left(\theta(y)+a e^{-\theta(y) \tau}+\lambda\right)^{-1} \hat{F}(\theta(y)) \theta^{\prime}(y) d y\right),
\end{aligned}
$$

\footnotetext{
${ }^{1}$ The quantity $x_{0}$ denotes the larger real root of $s+a e^{-\tau s}+\lambda_{\min }=0$ if this equation has real roots (see Lemma 2.2); otherwise, $x_{0}$ denotes the real part of the first complex root as described by Lemma 2.1.

${ }^{2}$ Note that $\frac{d y}{d x}=\tau|a| e^{-\tau\left(x-\beta_{1}\right)}-1<0$ and then Eq. (2.10) really implies an inverse function for all $x \in \mathbb{R}$.
} 

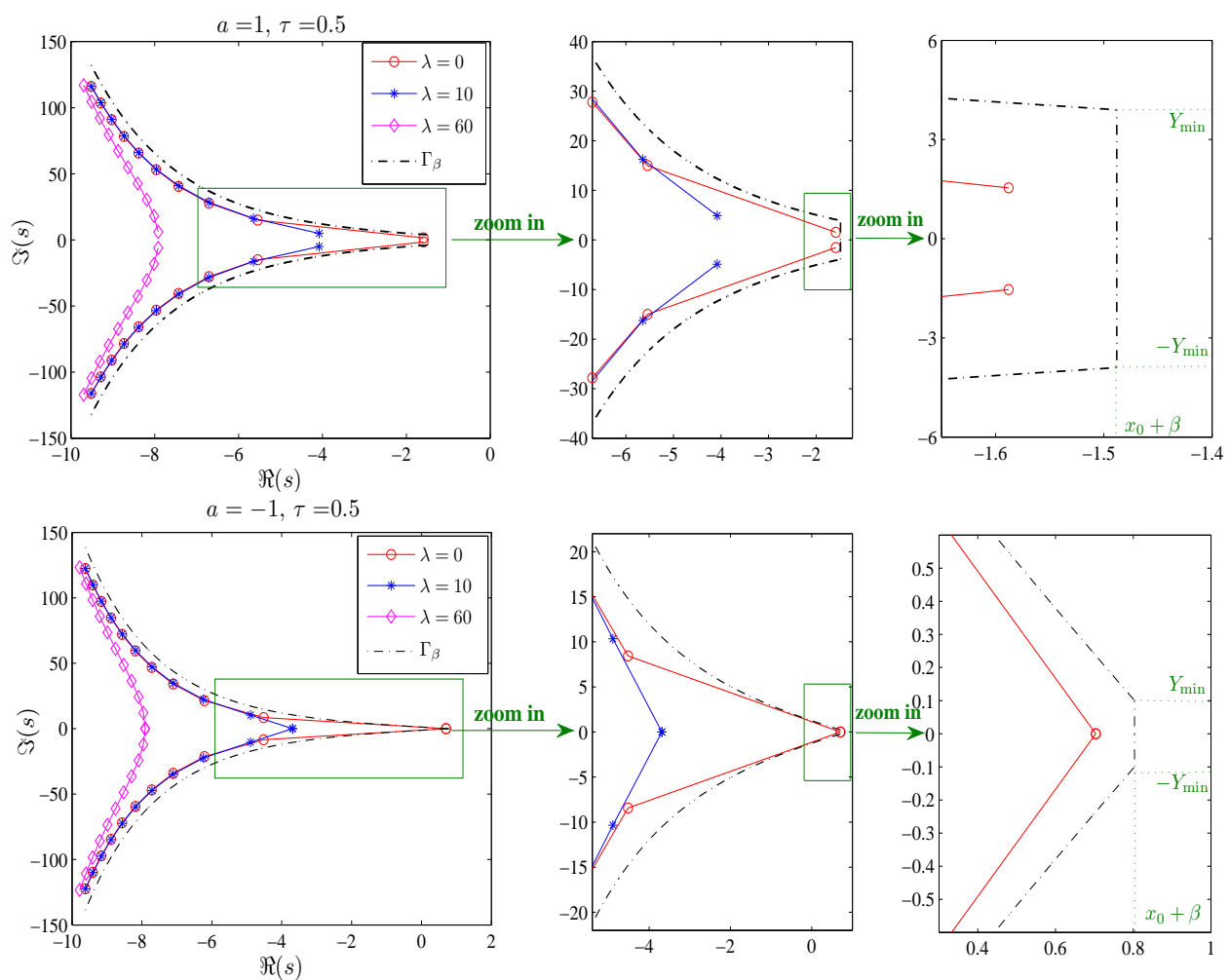

Figure 4: The roots of $s+a e^{-s \tau}+\lambda=0$ and the contour $\Gamma_{\boldsymbol{\beta}}$ defined by $(2.8)$ with $\boldsymbol{\beta}=(0.1,0.1)$. Top: $a=1$, for which no roots lie on the real axis; Bottom: $a=-1$, for which one root lies on the real axis.

where we have used the symmetry of $\theta(y)$ with respect to the real axis. From (2.9), we can divide the second integral in (2.11) into two parts

$$
\begin{aligned}
& \int_{0}^{\infty} e^{\theta(y) t}\left(\theta(y)+a e^{-\theta(y) \tau}+\lambda\right)^{-1} \hat{F}(\theta(y)) \theta^{\prime}(y) d y \\
= & i e^{\left(x_{0}+\beta_{0}\right) t} \int_{0}^{Y_{\min }} e^{i y t} \widetilde{F}_{1}(y) d y+\int_{Y_{\min }}^{\infty} e^{i y t} \widetilde{F}_{2}(t, y) d y,
\end{aligned}
$$

where the functions $\widetilde{F}_{1}(y)$ and $\widetilde{F}_{2}(t, y)$ are defined by

$$
\begin{aligned}
& \widetilde{F}_{1}(y)=\left(x_{0}+\beta_{0}+i y+a e^{-\left(x_{0}+\beta_{0}+i y\right) \tau}+\lambda\right)^{-1} \hat{F}\left(x_{0}+\beta_{0}+i y\right), \\
& \widetilde{F}_{2}(t, y)=e^{t X(y)}\left(X(y)+i y+a e^{-(X(y)+i y) \tau}+\lambda\right)^{-1} \hat{F}(X(y)+i y)\left(X^{\prime}(y)+i\right) .
\end{aligned}
$$

The function $X^{\prime}(y)$ appearing in the last integral can be expressed as a function of $X(y)$

$$
X^{\prime}(y)=-\frac{1}{|a| \tau e^{-\tau\left(X(y)-\beta_{1}\right)}+1} .
$$

For numerical computation, we need to truncate the integral $\int_{Y_{\min }}^{\infty} e^{i y t} \widetilde{F}_{2}(t, y) d y$ in (2.12) to a finite integral. For prescribed tolerance $\epsilon$, we have following result. 
Theorem 2.1. Let $\Gamma_{\beta}$ be a contour defined by (2.8) (or equivalently by (2.9)) such that all the roots of $s+a^{-s \tau}+\lambda=0$ lie on the left of $\Gamma_{\beta}$ for all $\lambda \geq \lambda_{\min }$. Then, for all $t \geq \Delta t$ it holds that

$$
\left|u(t)-\frac{1}{\pi} \mathfrak{I}\left(\int_{0}^{Y_{\max }} e^{\theta(y) t}\left(\theta(y)+a e^{-\theta(y) \tau}+\lambda\right)^{-1} \hat{F}(\theta(y)) \theta^{\prime}(y) d y\right)\right| \leq \epsilon,
$$

provided $Y_{\max }=\beta_{0}+|a| e^{-\tau\left(X_{\min }-\beta_{1}\right)}-\left(X_{\min }-\beta_{1}\right)$ and $X_{\min }$ satisfies

$$
X_{\min }<0, \quad \max _{x \leq X_{\min }}|\hat{F}(x+i y(x))|\left(\max _{x \leq X_{\min }} \frac{|a| \tau e^{\tau \beta_{1}}+e^{\tau x}}{\left(\beta_{0}+\beta_{1}-x\right) e^{\tau x}+|a|\left(e^{\tau \beta_{1}}-1\right)}\right) \frac{e^{\Delta t X_{\min }}}{\Delta t} \leq \frac{\epsilon}{4}
$$

where $y(x)=\beta_{0}+|a| e^{-\tau\left(x-\beta_{1}\right)}-\left(x-\beta_{1}\right)$.

Proof. Clearly, it suffices to prove

$$
\int_{Y_{\max }}^{\infty} e^{t X(y)}\left|\frac{X^{\prime}(y)+i}{X(y)+i y+a e^{-\tau(X(y)+i y)}+\lambda}\right| d y \leq \frac{\epsilon}{4 \max _{x \leq X_{\min }}|\hat{F}(x+i y(x))|}
$$

Since

$$
\left|X(y)+i y+a e^{-(X(y)+i y) \tau}+\lambda\right| \geq\left|y-a e^{-X(y) \tau} \sin (y \tau)\right|,
$$

and $y>|a| e^{-\tau X(y)}$ for $y \geq Y_{\max }$, it holds that

$$
\begin{aligned}
& \int_{Y_{\max }}^{\infty} e^{t X(y)}\left|\frac{X^{\prime}(y)+i}{X(y)+i y+a e^{-\tau(X(y)+i y)}+\lambda}\right| d y \\
\leq & \int_{Y_{\max }}^{\infty} e^{t X(y)} \frac{\sqrt{1+\left(X^{\prime}(y)\right)^{2}}}{y-|a| e^{-\tau X(y)}} d y \leq \int_{Y_{\max }}^{\infty} e^{t X(y)} \frac{1+\left|X^{\prime}(y)\right|}{y-|a| e^{-\tau X(y)}} d y .
\end{aligned}
$$

This, together with

$$
\frac{1+\left|X^{\prime}(y)\right|}{y-|a| e^{-\tau X(y)}}=\frac{1+\frac{1}{|a| \tau e^{-\tau\left(X(y)-\beta_{1}\right)+1}}}{y-|a| e^{-\tau X(y)}} \leq \frac{2}{y-|a| e^{-\tau X(y)}} \quad \text { for } y \leq Y_{\max },
$$

gives

$$
\int_{Y_{\max }}^{\infty} e^{t X(y)}\left|\frac{X^{\prime}(y)+i}{X(y)+i y+a e^{-\tau(X(y)+i y)}+\lambda}\right| d y \leq 2 \int_{Y_{\max }}^{\infty} \frac{e^{t X(y)}}{y-|a| e^{-\tau X(y)}} d y .
$$


Now, by letting $x=X(y)$ and by using $y=\beta_{0}+|a| e^{-\tau\left(X(y)-\beta_{1}\right)}-\left(X(y)-\beta_{1}\right)$ we can rewrite the integral in the right hand-side of (2.17) as

$$
\begin{aligned}
& \int_{Y_{\max }}^{\infty} \frac{e^{t X(y)}}{y-|a| e^{-\tau X(y)}} d y \\
= & \int_{X_{\min }}^{-\infty} \frac{e^{t x}}{\beta_{0}+\beta_{1}-x+|a| e^{-\tau x}\left(e^{\tau \beta_{1}}-1\right)}\left(-|a| \tau e^{-\tau\left(x-\beta_{1}\right)}-1\right) d x \\
= & \int_{-\infty}^{X_{\min }} \frac{e^{t x}\left(|a| \tau e^{\tau \beta_{1}}+e^{\tau x}\right)}{\left(\beta_{0}+\beta_{1}-x\right) e^{\tau x}+|a|\left(e^{\tau \beta_{1}}-1\right)} d x \\
\leq & \left(\max _{x \leq X_{\min }} \frac{|a| \tau e^{\tau \beta_{1}}+e^{\tau x}}{\left(\beta_{0}+\beta_{1}-x\right) e^{\tau x}+|a|\left(e^{\tau \beta_{1}}-1\right)}\right) \frac{e^{t X_{\min }}}{t} .
\end{aligned}
$$

Substituting (2.18) into (2.17) and then by using (2.16), we get (2.15a) if (2.15b) holds. The proof is completed.

\subsection{Summary}

For a suitably large quantity $Y_{\max }$, to get an approximation (with truncation error $\epsilon$ ) of the solution $u(\mathbf{x}, t)$ of the delay problem (1.2), Theorem 2.1 implies that it is sufficient to consider the following truncated contour integral

$$
u(\mathbf{x}, t) \approx \frac{1}{\pi} \mathfrak{I}\left(i e^{\left(x_{0}+\beta_{0}\right) t} \int_{0}^{Y_{\min }} e^{i y t} \hat{F}_{1}(\mathbf{x}, y) d y+\int_{Y_{\min }}^{Y_{\max }} e^{i y t} \hat{F}_{2}(\mathbf{x}, t, y) d y\right),
$$

where $\hat{F}_{1}(\mathbf{x}, y)$ and $\hat{F}_{2}(\mathbf{x}, y)$ are given by

$$
\begin{aligned}
& \hat{F}_{1}(\mathbf{x}, y)=\left(\left[x_{0}+\beta_{0}+i y+a e^{-\left(x_{0}+\beta_{0}+i y\right) \tau}\right] I-\Delta\right)^{-1} \hat{F}\left(\mathbf{x}, x_{0}+\beta_{0}+i y\right), \\
& \hat{F}_{2}(\mathbf{x}, t, y)=e^{t X(y)}\left(\left[X(y)+i y+a e^{-(X(y)+i y) \tau}\right] I-\Delta\right)^{-1} \hat{F}(\mathbf{x}, X(y)+i y)\left(X^{\prime}(y)+i\right), \\
& \hat{F}(\mathbf{x}, s)=u_{0}(\mathbf{x}, 0)-a \int_{0}^{\tau} e^{-s r} u_{0}(\mathbf{x}, r-\tau) d r+\hat{f}(\mathbf{x}, s), \quad \hat{f}(\mathbf{x}, s)=\int_{0}^{\infty} e^{t s} f(\mathbf{x}, t) d t .
\end{aligned}
$$

We note that both $\hat{F}_{1}(\mathbf{x}, y)$ and $\hat{F}_{2}(\mathbf{x}, y)$ contain $\hat{F}(\mathbf{x}, s)$, in which an integral $\int_{0}^{\tau} e^{-s r} u_{0}(\mathbf{x}, r-$ $\tau) d r$ is involved. For $\hat{F}_{2}$, the real part of the complex number $s$ becomes negative when $s$ approaches the ends of the contour $\Gamma_{\beta}$; for $\hat{F}_{1}$, the real part of $s$ is $x_{0}+\beta_{0}$, which can be negative, too. To avoid floating overflow in computation, it is necessary to make some modifications. The idea is to split each integral in (2.19) into two parts:

$$
\begin{aligned}
& \int_{0}^{Y_{\min }} e^{i y t} \hat{F}_{1}(\mathbf{x}, y) d y=\int_{0}^{Y_{\min }} e^{i y t} \hat{F}_{11}(\mathbf{x}, y)-a e^{-\left(x_{0}+\beta\right) \tau} \int_{0}^{Y_{\min }} e^{i y(t-\tau)} \hat{F}_{12}(\mathbf{x}, y), \\
& \int_{Y_{\min }}^{Y_{\max }} e^{i y t} \hat{F}_{2}(\mathbf{x}, y) d y=\int_{Y_{\min }}^{Y_{\max }} e^{i y t} \hat{F}_{21}(\mathbf{x}, y)-a \int_{Y_{\min }}^{Y_{\max }} e^{i y(t-\tau)} \hat{F}_{22}(\mathbf{x}, y)
\end{aligned}
$$


where $\hat{F}_{11}(\mathbf{x}, y), \hat{F}_{12}(\mathbf{x}, y), \hat{F}_{21}(\mathbf{x}, y)$ and $\hat{F}_{22}(\mathbf{x}, y)$ are given by

$$
\begin{aligned}
\hat{F}_{11}(\mathbf{x}, y) & =\left(\left[x_{0}+\beta_{0}+i y+a e^{-\left(x_{0}+\beta_{0}+i y\right) \tau}\right] I-\Delta\right)^{-1}\left(u_{0}(\mathbf{x}, 0)+\hat{f}\left(\mathbf{x}, x_{0}+\beta_{0}+i y\right)\right), \\
\hat{F}_{12}(\mathbf{x}, y) & =\left(\left[x_{0}+\beta_{0}+i y+a e^{-\left(x_{0}+\beta_{0}+i y\right) \tau}\right] I-\Delta\right)^{-1} \int_{0}^{\tau} e^{\left(x_{0}+\beta_{0}+i y\right)(\tau-r)} u_{0}(\mathbf{x}, r-\tau) d r, \\
\hat{F}_{21}(\mathbf{x}, y) & =e^{t X(y)}\left(X^{\prime}(y)+i\right)([X(y)+i y \\
& \left.\left.+a e^{-(X(y)+i y) \tau}\right] I-\Delta\right)^{-1}\left[u_{0}(\mathbf{x}, 0)+\hat{f}(\mathbf{x}, X(y)+i y)\right], \\
\hat{F}_{22}(\mathbf{x}, y)=e^{(t-\tau) X(y)}\left(X^{\prime}(y)+i\right)([X(y)+i y & \left.\left.+a e^{-(X(y)+i y) \tau}\right] I-\Delta\right)^{-1} \int_{0}^{\tau} e^{(X(y)+i y)(\tau-r)} u_{0}(\mathbf{x}, r-\tau) d r .
\end{aligned}
$$

\section{Filon-Clenshaw-Curtis quadrature}

We are now in a position to study how to discretize the four integrals in right hand-sides of (2.22a) and (2.22b). These are highly-oscillatory integrals and can not be computed by usual numerical methods, such as the Gauss-Legendre method; see $[12,15,16]$ for explanations. Here, we study how to use the Filon-Clenshaw-Curtis quadrature $[8,9,31]$ to compute these integrals. For the two integrals in (2.22a), we can directly apply the FCC quadrature, because $Y_{\min }$ is only a moderate quantity. For the other two integrals in (2.22b), we however need to divide the interval $\left[Y_{\min }, Y_{\max }\right]$ into subintervals and then apply the FCC quadrature to each subinterval. In what follows, we focus on the FCC quadrature for the integrals in (2.22b) and all the results can be directly applied to (2.22a). Moreover, for the two integrals in the right hand-side of (2.22b), $\int_{Y_{\min }}^{Y_{\max }} e^{i y t} \hat{F}_{21}(\mathbf{x}, y)$ and $\int_{Y_{\min }}^{Y_{\max }} e^{i y(t-\tau)} \hat{F}_{22}(\mathbf{x}, y)$, it is sufficient to introduce the FCC quadrature for one of them. We shall consider the latter and the results can be directly generalized to the former.

Let $B \geq 2$ be an integer and $J_{\max }=1+\left[\log _{B}\left(Y_{\max }-Y_{\min }\right)\right]$, where for any real number $v$ we denote by $[v]$ the integer part of $v$. Then, with another integer $J_{\min }>0$, we divide the interval $\left[Y_{\min }, Y_{\max }\right]$ into $J_{\max }+J_{\min }+2$ subintervals, as

$$
\left[Y_{\min }, Y_{\max }\right]=\bigcup_{j=-J_{\min }-1}^{J_{\max }}\left[Y_{j}, Y_{j+1}\right], \text { with } Y_{j}= \begin{cases}Y_{\min }, & j=-J_{\min }-1, \\ Y_{\min }+B^{j}, & -J_{\min } \leq J \leq J_{\max } \\ Y_{\max }, & j=J_{\max }+1\end{cases}
$$

The integral $\int_{Y_{\min }}^{Y_{\max }} e^{i y(t-\tau)} \hat{F}_{22}(\mathbf{x}, y) d y$ therefore can be rewritten as:

$$
\int_{Y_{\min }}^{Y_{\max }} e^{i y(t-\tau)} \hat{F}_{22}(\mathbf{x}, y) d y=\sum_{j=-J_{\min }-1}^{J_{\max }} \mathscr{J}_{j}, \quad \text { with } \mathscr{J}_{j}=: \int_{Y_{j}}^{Y_{j+1}} e^{i y(t-\tau)} \hat{F}_{22}(\mathbf{x}, y) d y .
$$


For each $\mathscr{J}_{j}$, we need to compute an inner integral for each $y$ :

$$
\int_{0}^{\tau} e^{s(\tau-r)} u_{0}(\mathbf{x}, r-\tau) d r, \quad \text { with } s=X(y)+i y .
$$

The integral in (3.3) is different from $\mathscr{J}_{j}$ : for the former the parameter $s$ is a complex quantity, while for the latter the parameter $i(t-\tau)$ is purely imaginary. For the integral in (3.3), even though the parameter $s$ has negative real part (and therefore the exponential term $e^{s(\tau-r)}$ provides rapid decaying for the whole integrand), it still can not be efficiently computed by the Gauss-Legendre method. To illustrate this, we consider the model problem (1.1), where $u_{0}(\mathbf{x}, r-\tau)=\cos \left(\frac{\pi}{2 \tau}(r-\tau)\right)$. For $y$ varies from $Y_{\min }$ to $5 \times 10^{4}$, we plot in Fig. 5 on the top row the error of the Gauss-Legendre method for the following integral ${ }^{3}$

$$
\int_{0}^{\tau} e^{s(\tau-r)} \cos \left(\frac{\pi}{2 \tau}(r-\tau)\right) d r, \text { with } s=X(y)+i y
$$

where $X(y)$ denotes the inverse function implied by $y=\beta_{0}+|a| e^{-\tau\left(x-\beta_{1}\right)}-\left(x-\beta_{1}\right)$ with $a=\frac{\pi}{2 \tau}$. We consider two values of $\tau: \tau=0.05$ and $\tau=1$. For both values, we choose $\beta_{0}=2$ and $\beta_{1}=0.2$. The quantity $Y_{\min }$ is determined by $Y_{\min }=\beta_{1}+|a| e^{-\tau\left(x_{0}+\beta_{0}-\beta_{1}\right)}-x_{0}$, where $x_{0}$ denotes the maximal real part of the roots of $s+a e^{-\tau s}=0$. In each subfigure, we plot the error for 4 values of $M$, the number of the Gauss-Legendre nodes. We see that, as $y$ becomes large, i.e., $s$ moves to the ends of the contour $\Gamma_{\beta}$, the error of the Gauss-Legendre method grows rapidly to a value of order $\mathscr{O}\left(10^{-1}\right)$. Increasing $M$ does not provide essential improvement. For comparisons, the error of the FCC quadrature which will be introduced in detail in the following is also shown in Fig. 5 on the bottom row. Apparently, for integral containing an exponential term $e^{z y}$ with $z \in \mathbb{C}$ and $\mathfrak{I}(z)$ large, the FCC quadrature is superior. For the FCC quadrature, the moments are computed by the two-phase procedure, where the switch point between the two phases is determined by an error tolerance $\delta=10^{-12}$ (more details are given in Section 3.2.2).

To describe the FCC quadrature, we consider the integrals of the following two forms:

$$
\mathscr{J}[k, F]:=\int_{-1}^{1} e^{i k y} F(y) d y, \quad \mathscr{I}[z, F]:=\int_{-1}^{1} e^{z(1-y)} F(y) d y,
$$

which respectively correspond to the integrals $\mathscr{J}_{j}$ and the inner integral (3.3) after a proper change of variable. For example, for $\mathscr{J}_{j}$ by letting $y=0.5\left(Y_{j+1}+Y_{j}\right)+0.5\left(Y_{j+1}-Y_{j}\right) \tilde{y}$ we have

$$
\begin{array}{r}
0.5\left(Y_{j+1}-Y_{j}\right) e^{i(t-\tau)\left(Y_{j+1}+Y_{j}\right) / 2} \int_{-1}^{1} \tilde{y} e^{i(t-\tau)\left(Y_{j+1}-Y_{j}\right) / 2} \hat{F}_{22} \\
\quad \cdot\left(\mathbf{x}, 0.5\left(Y_{j+1}+Y_{j}\right)+0.5\left(Y_{j+1}-Y_{j}\right) \tilde{y}\right) d \tilde{y} .
\end{array}
$$

${ }^{3}$ The exact result of this integral is $\left(a e^{s \tau}-s\right) /\left(s^{2}+a^{2}\right)$ with $a=\pi /(2 \tau)$. 

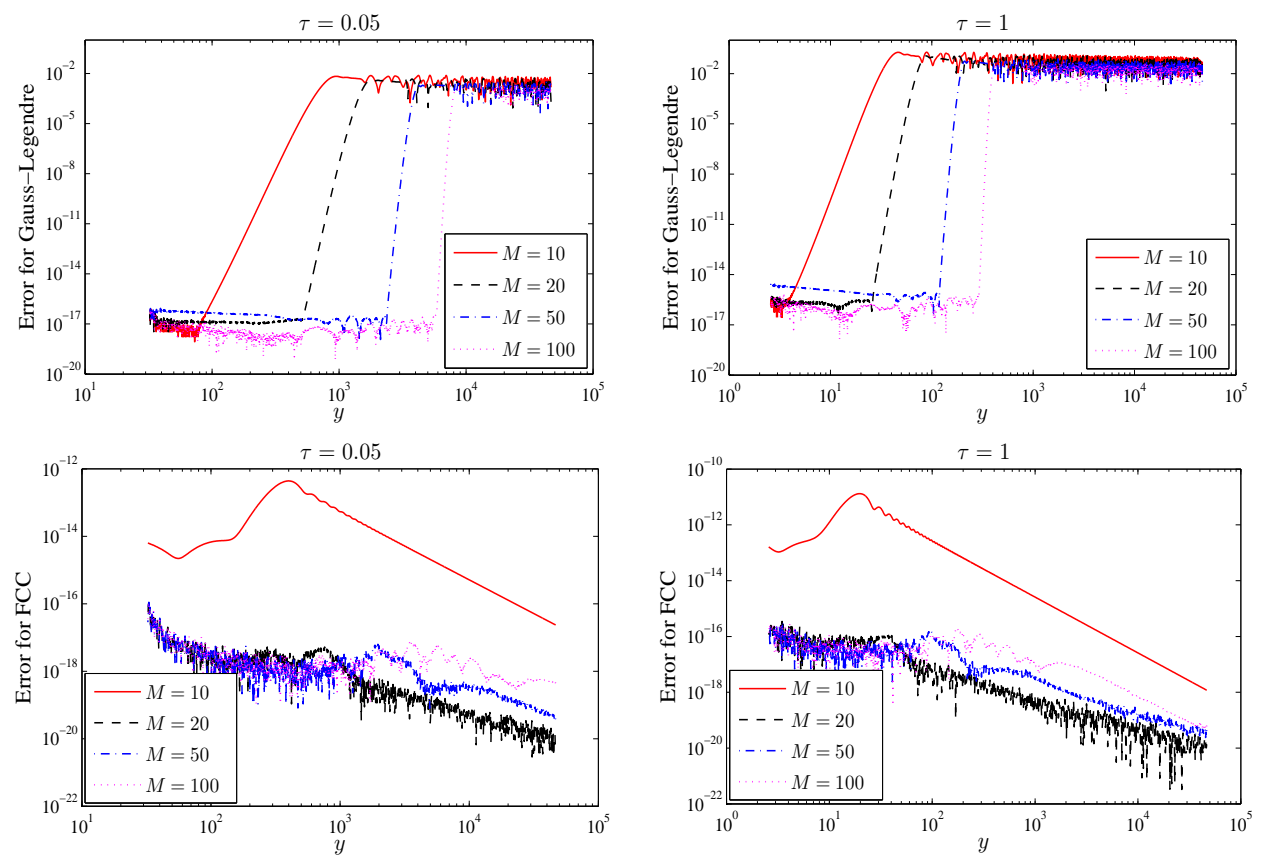

Figure 5: Errors of the $M$-node Gauss-Legendre method (top row) and the FCC quadrature (bottom row) for integral (3.4). The contour $\Gamma_{\beta}$ is determined by (2.8) with $\beta_{0}=2$ and $\beta_{1}=0.2$.

Then, by letting $k=(t-\tau)\left(Y_{j+1}-Y_{j}\right) / 2$ and $F(y)=\hat{F}_{22}\left(\mathbf{x}, 0.5\left(Y_{j+1}+Y_{j}\right)+0.5\left(Y_{j+1}-Y_{j}\right) y\right)$, we have $\mathscr{J}_{j}=0.5\left(Y_{j+1}-Y_{j}\right) e^{i(t-\tau)\left(Y_{j+1}+Y_{j}\right) / 2} \mathscr{J}[k, F]$.

Let $Q_{M} F$ denotes the interpolate of $F$ by the first-type Chebyshev polynomials $\left\{T_{m}(y)=\right.$ $\left.\cos \left(m \cos ^{-1}(y)\right)\right\}_{m=0}^{M}$ at the Clenshaw-Curtis points $y_{m, M}=\cos \left(\frac{m \pi}{M}\right)($ with $m=0,1, \cdots, M)$

$$
Q_{M} F(y)=\sum_{m=0}^{M} \prime \prime \alpha_{m, M}(F) T_{m}(y), \quad \alpha_{m, M}(F)=\frac{2}{M} \sum_{j=0}^{M}{ }^{\prime \prime} \cos \left(\frac{j m \pi}{M}\right) F\left(y_{j, M}\right) .
$$

Here and hereafter, $\sum^{\prime \prime}$ means that the first and the last terms in the sum are to be halved. Replacing $F$ in (3.5) by $Q_{M} F$ gives the formula of the FCC quadrature:

$$
\begin{array}{r}
\mathscr{J}_{M}[k, F]=\int_{-1}^{1} e^{i k y} Q_{M} F(y) d y=\sum_{m=0}^{M} " \prime \alpha_{m, M}(F) \eta_{m}, \\
\text { with } \eta_{m}=\int_{-1}^{1} e^{i k y} T_{m}(y) d y, \\
\mathscr{I}_{M}[z, F]=\int_{-1}^{1} e^{z(1-y)} Q_{M} F(y) d y=\sum_{m=0}^{M} \alpha_{m, M}(F) \omega_{m}, \\
\text { with } \omega_{m}=\int_{-1}^{1} e^{z(1-y)} T_{m}(y) d y .
\end{array}
$$


The FCC quadrature coefficients $\left\{\alpha_{m, M}\right\}_{m=0}^{M}$ can be written as $\mathbf{a}_{M}(F)=\mathbf{C}_{M} \mathbf{F}_{M}$, where

$$
\begin{aligned}
& \mathbf{F}_{M}=\left(\frac{F\left(y_{0, M}\right)}{2}, F\left(y_{1, M}\right), \cdots, F\left(y_{M-1, M}\right), \frac{F\left(y_{M, M}\right)}{2}\right)^{\top}, \\
& \mathbf{C}_{M}=\left(C_{m, j}\right)_{m, j=0}^{M} \text { with } C_{m, j}=\frac{2}{M} \cos \left(\frac{j m \pi}{M}\right) .
\end{aligned}
$$

The matrix-vector multiplication $\mathbf{C}_{M} \mathbf{F}_{M}$ is the so-called discrete cosine transform of type I [7, Section 4.7.25] and it can be computed by FFT in $\mathscr{O}(M \log M)$ operations. Let

$$
\eta_{M}=\left[\eta_{0} / 2, \eta_{1}, \cdots, \eta_{M-1}, \eta_{M} / 2\right]^{\top}, \quad \omega_{M}=\left[\omega_{0} / 2, \omega_{1}, \cdots, \omega_{M-1}, \omega_{M} / 2\right]^{\top} .
$$

Then, by using the symmetry of $\mathbf{C}_{M}$ we can represent $\mathscr{J}_{M}[k, F]$ and $\mathscr{I}_{M}[z, F]$ in (3.6) as

$$
\begin{aligned}
& \mathscr{J}_{M}[k, F]=\boldsymbol{\eta}_{M}^{\top} \mathbf{a}_{M}(F)=\boldsymbol{\eta}_{M}^{\top} \mathbf{C}_{M} \mathbf{F}_{M}=\left(\mathbf{C}_{M} \boldsymbol{\eta}_{M}\right)^{\top} \mathbf{F}_{M}, \\
& \mathscr{I}_{M}[z, F]=\omega_{M}^{\top} \mathbf{a}_{M}(F)=\omega_{M}^{\top} \mathbf{C}_{M} \mathbf{F}_{M}=\left(\mathbf{C}_{M} \boldsymbol{\omega}_{M}\right)^{\top} \mathbf{F}_{M} .
\end{aligned}
$$

This implies that if we precompute $\mathrm{C}_{M} \boldsymbol{\eta}_{M}$ and $\mathrm{C}_{M} \boldsymbol{\omega}_{M}$, we can apply the FCC quadrature to many different functions $F$.

\subsection{Error bounds of the FCC quadrature}

In this subsection, we present the error bounds for the FCC quadratures $\mathscr{J}_{M}[k, F]$ and $\mathscr{I}_{M}[\mathcal{z}, F]$.

Theorem 3.1. Let $c=0,1,2$ and $v \geq v_{0}>\max \{0.5, \rho(c)\}$. Then, for the integral $\mathscr{J}[k, F]$ which is approximated by the FCC quadrature $\mathscr{J}_{M}[k, F]$ it holds for all $k \in \mathbb{R} \backslash\{0\}$ that

$$
\left|\mathscr{J}[k, F]-\mathscr{J}_{M}[k, F]\right| \leq C_{v_{0}} \min \left\{1,|k|^{-c}\right\} M^{-(v-\rho(c))}\|F\|_{\mathbb{H}^{v}},
$$

where $C_{v_{0}}>0$ is a quantity depending on $v_{0}$ only, $\rho(0)=0, \rho(1)=1, \rho(2)=7 / 2$ and $\|F\|_{\mathbb{H}^{v}}$ denotes the Sobolev norm defined by

$$
\|F\|_{\mathbb{H}^{v}}^{2}=\left|\bar{F}_{0}\right|^{2}+\sum_{l \neq 0}|l|^{2 v}\left|\bar{F}_{l}\right|^{2} \text {, with } \bar{F}_{l}=\frac{1}{\pi} \int_{0}^{\pi} F(\cos (\phi)) e^{i l \phi} d \phi .
$$

Proof. The error bound (3.7) can be found in [8].

This error bound implies that for all $k$, the FCC quadrature is efficient and uniformly convergent for computing the integral $\mathscr{J}[k, F]$ under the assumption that $F$ possess suitable regularity. Other error bounds which treat the cases that $F$ has algebraic singularities and limited regularity can be found in [9] and [31], respectively. To control the length of the paper, we shall not pursue this here. For the integral $\mathscr{I}[z, F]$, by performing a similar analysis given in [8] we have the following error bounds for the FCC quadrature $\mathscr{I}_{M}[z, F]$. 
Theorem 3.2. For $c \in\{0,1,2\}$ and for all $v \geq v_{0}>\max \{0.5, \rho(c)\}$, it holds that

$$
\left|\mathscr{I}[z, F]-\mathscr{I}_{M}[z, F]\right| \leq \begin{cases}C_{v_{0}} \gamma(c) M^{-v}\|F\|_{\mathbb{H}_{v}}, & \text { if }|z| \leq 1, \\ C_{v_{0}} \gamma(c)|z|^{-c} M^{-(v-\rho(c))}\|F\|_{\mathbb{H}_{v}}, & \text { if }|z|>1,\end{cases}
$$

where $C_{v_{0}}$ is a quantity depending on $v_{0}$ only and the constants $\gamma(c)$ and $\rho(c)$ are defined by

$$
\gamma(c)=\left\{\begin{array}{ll}
\sqrt{\frac{1}{2} \int_{0}^{\pi} \sin ^{2}(\phi) e^{2 \Re(z)(1-\cos (\phi)) d \phi}}, & c=0, \\
\sqrt{\frac{1}{2} \int_{0}^{\pi} e^{2 \Re(z)(1-\cos (\phi)) d \phi},} & c=1, \\
\max \left\{1, e^{2 \Re(z)}\right\}+\int_{0}^{\pi} e^{\Re(z)(1-\cos (\phi))} d \phi, & c=2,
\end{array} \quad \rho(c)= \begin{cases}0, & c=0, \\
1, & c=1, \\
\frac{7}{2}, & c=2 .\end{cases}\right.
$$

Remark 3.1. From (3.8), we see that the accuracy of the FCC quadrature improves as the module of the complex number $z$ increases, since a term $|z|^{-c}$ is contained in the error bound. This property can be seen in Fig. 5 on the bottom row. For the purely imaginary case, i.e., an exponential term $e^{i k y}$ instead of $e^{z y}$ is contained in the integrand, such a welcome property is known long in the area of highly-oscillatory quadrature $[8,9,15,16$, $31]$.

\subsection{Stable computation of the FCC quadrature moments $\left\{\eta_{m}\right\}$ and $\left\{\omega_{m}\right\}$}

To use the FCC quadratures in practice, it remains to address how to get the FCC quadrature moments $\left\{\eta_{m}\right\}_{m=0}^{M}$ and $\left\{\omega_{m}\right\}_{m=0}^{M}$.

\subsubsection{The computation of $\left\{\eta_{m}\right\}$}

For the integral $\mathscr{J}[k, F]$ in which a purely imaginary parameter is involved, the computation of the FCC quadrature moments $\left\{\eta_{m}\right\}$ is carefully studied in [8]. Here, we revisit the main procedure for completeness. Some notations are also useful to describe the computation of $\left\{\omega_{m}\right\}$ in the next subsection. Recall that the $m$-th Chebyshev polynomial of the second type is defined by $\tilde{T}_{m}(y)=\frac{1}{1+m} T_{m+1}^{\prime}(y)$, where $\left\{T_{m}(y)=\cos (m \arccos (y))\right\}_{m \geq 0}$ are the Chebyshev polynomials of the first type. These two types of Chebyshev polynomials are related by $2 T_{m}=\tilde{T}_{m}-\tilde{T}_{m-2}$ for all $m \geq 2$. Since $\eta_{m}=\int_{-1}^{1} e^{i k y} T_{m}(y) d y$, we have

$$
2 \eta_{m}=\int_{-1}^{1}\left(2 T_{m}(y)\right) e^{i k y} d y=\tilde{\eta}_{m+1}-\tilde{\eta}_{m-1} \quad \text { for } m \geq 2,
$$

where

$$
\tilde{\eta}_{m}=\int_{-1}^{1} \tilde{T}_{m-1}(y) e^{i k y} d y=\frac{1}{m} \int_{-1}^{1} T_{m}^{\prime}(y) e^{i k y} d y .
$$

On the other hand, integrating the formula $\int_{-1}^{1} e^{i k y} T_{m}(y) d y$ by part for $\eta_{m}$ gives

$$
\eta_{0}=\mu_{0}, \quad \eta_{m}=\mu_{m}-\frac{m}{i k} \tilde{\eta}_{m} \text {, with } \mu_{m}=\left.\frac{1}{i k} T_{m}(y) e^{i k y}\right|_{y=-1} ^{y=1}=\frac{e^{i k}-(-1)^{m} e^{-i k}}{i k} \text {. }
$$



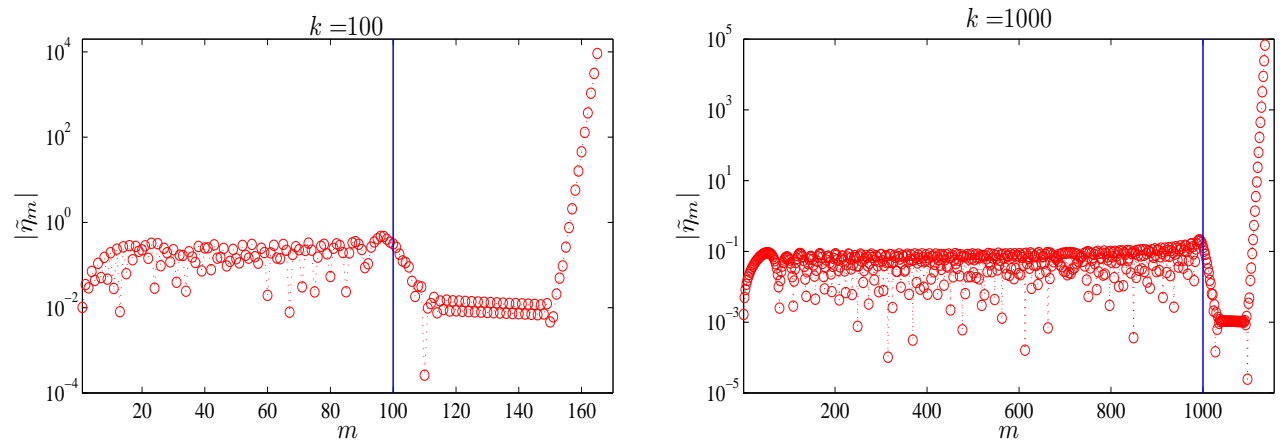

Figure 6: Computing the moments $\left\{\tilde{\eta}_{m}\right\}_{m \geq 1}$ by directly forwarding the three-term recurrence (3.11) is unstable in general, but for $m \leq k$ it seems that the computation is stable.

Hence, $2 \mu_{m}-\frac{2 m}{i k} \tilde{\eta}_{m}=\tilde{\eta}_{m+1}-\tilde{\eta}_{m-1}$ for $m \geq 2$. Since $T_{1}^{\prime}(y)=1$ and $T_{2}^{\prime}(y)=4 y$, from (3.9) we get

$$
\tilde{\eta}_{1}=\int_{-1}^{1} e^{i k y} d y=\mu_{0}, \quad \tilde{\eta}_{2}=2 \int_{-1}^{1} y e^{i k y} d y=2 \mu_{1}-\frac{2}{i k} \mu_{0} .
$$

Now, we can write down the recurrence relation for the quantities $\left\{\tilde{\eta}_{m}\right\}_{m \geq 1}$ as

$$
\tilde{\eta}_{1}=\mu_{0}, \quad \tilde{\eta}_{2}=2 \mu_{1}-\frac{2}{i k} \mu_{0}, \quad \tilde{\eta}_{m+1}+\frac{2 m}{i k} \tilde{\eta}_{m}-\tilde{\eta}_{m-1}=2 \mu_{m} \quad \text { for } m \geq 2 .
$$

With $\left\{\tilde{\eta}_{m}\right\}_{m \geq 1}$, the moments $\left\{\eta_{m}\right\}_{m \geq 0}$ for the FCC quadrature $\mathscr{J}_{M}[k, F]$ can be fixed as

$$
\eta_{0}=\mu_{0}, \quad \eta_{m}=\mu_{m}-\frac{m}{i k} \tilde{\eta}_{m} \quad \text { for } m \geq 1 .
$$

Since the initial conditions $\tilde{\eta}_{1}$ and $\tilde{\eta}_{2}$ are given, the required solution $\left\{\tilde{\eta}_{m}\right\}_{m \geq 3}$ is in theory completely specified. But it may not be possible to compute the desired solution by simply forwarding the three-term recurrence (3.11), owing to a rapid increasing of rounding error as illustrated in Fig. 6. The plots indicate that the computation of $\left\{\tilde{\eta}_{m}\right\}_{m \geq 1}$ is unstable, because for given $k$ it should hold asymptotically that $\left|\tilde{\eta}_{m}\right| \rightarrow 0$ as $m \rightarrow \infty^{4}$.

The idea for stably computing $\left\{\tilde{\eta}_{m}\right\}_{m \geq 1}$ proposed in [8] is based on a two-phase procedure. In the first phase, we compute $\left\{\tilde{\eta}_{m}\right\}_{m=1}^{m_{0}}$ by directly forwarding (3.11). In the second phase, we compute the remainder $\{\tilde{\eta}\}_{m=m_{0}+1}^{M}$ by solving a tri-diagonal linear system

$$
\underbrace{\left(\begin{array}{ccccc}
\frac{2\left(m_{0}+1\right)}{i k} & 1 & & & \\
-1 & \frac{2\left(m_{0}+2\right)}{i k} & 1 & & \\
& \ddots & \ddots & \ddots & \\
& & -1 & \frac{2(K-2)}{i k} & 1 \\
& & & -1 & \frac{2(K-1)}{i k}
\end{array}\right)}_{:=A_{m_{0}, K}(k)}\left(\begin{array}{c}
\tilde{\eta}_{m_{0}+1} \\
\tilde{\eta}_{m_{0}+2} \\
\vdots \\
\tilde{\eta}_{K-2} \\
\tilde{\eta}_{K-1}
\end{array}\right)=\left(\begin{array}{c}
2 \mu_{m_{0}+1}+\tilde{\eta}_{m_{0}} \\
2 \mu_{m_{0}+2} \\
\vdots \\
2 \mu_{K-2} \\
2 \mu_{K-1}-\tilde{\eta}_{K}
\end{array}\right) \text {, }
$$
${ }^{4}$ To see this, we note that $\eta_{m}=\int_{-1}^{1} e^{i k y} T_{m}(y) d y \Rightarrow\left|\eta_{m}\right| \leq 2$ and this, together with (3.12) and $\left|\mu_{m}\right| \leq 2 /|k|$,
gives $\left|\tilde{\eta}_{m}\right| \leq(2+|k|) / m$. Hence, $\lim _{m \rightarrow \infty}\left|\tilde{\eta}_{m}\right|=0$. 
where $\tilde{\eta}_{m_{0}}$ is obtained from the first phase and the quantity $\tilde{\eta}_{K}$ is a priori unknown, but if we take $K$ sufficiently large it can be approximated accurately by an asymptotic expansion (see [8, Section 4]). If $M$ is small, we can compute all the moments $\{\tilde{\eta}\}_{m=2}^{M}$ in the first phase. It was proven in [8] that if $m_{0}=[k]+1$ the computation of the first phase is stable; see Fig. 6 for evidences. The proof for such a stability is based on the fact that for this choice of $m_{0}$ the coefficient matrix $A_{m_{0}, K}(k)$ is diagonally dominate (see [8, Section 5] for more details).

\subsubsection{The computation of $\left\{\omega_{m}\right\}$}

For the moments $\left\{\omega_{m}\right\}$ of the FCC quadrature $\mathscr{I}_{M}[z, F]$, we define

$$
\xi_{m}=\left.\frac{-1}{z} T_{m}(\tau) e^{z(1-\tau)}\right|_{-1} ^{1}=\frac{(-1)^{m} e^{2 z}-1}{z} \text { for } m \geq 0 .
$$

Then, similar to (3.12) the moments $\left\{\omega_{m}\right\}_{m \geq 0}$ can be determined by

$$
\omega_{0}=\xi_{0}, \quad \omega_{m}=\xi_{m}+\frac{m}{z} \tilde{\omega}_{m},
$$

where

$$
\tilde{\omega}_{m}=\int_{-1}^{1} \tilde{T}_{m-1}(y) e^{z(1-y)} d y=\frac{1}{m} \int_{-1}^{1} T_{m}^{\prime}(y) e^{z(1-y)} d y \quad \text { for } m \geq 1 .
$$

Similar to (3.11), these transformed moments $\left\{\tilde{\omega}_{m}\right\}_{m \geq 1}$ also satisfy a three-term recurrence, as

$$
\tilde{\omega}_{1}=\xi_{0}, \quad \tilde{\omega}_{2}=\frac{2}{z} \xi_{1}+2 \xi_{0}, \quad \tilde{\omega}_{m+1}-\frac{2 m}{z} \tilde{\omega}_{m}-\tilde{\omega}_{m-1}=2 \xi_{m} \quad \text { for } m \geq 2 .
$$

In general, the computation of the $\left\{\tilde{\omega}_{m}\right\}_{m=0}^{M}$ is also based on the two-phase procedure: in the first phase we compute $\left\{\tilde{\omega}_{m}\right\}_{m=0}^{m_{0}}$ by directly forwarding the recurrence (3.14) and then in the second phase we compute $\left\{\tilde{\omega}_{m}\right\}_{m_{0}+1}^{M}$ be solving a tri-diagonal linear system

$$
\underbrace{\left(\begin{array}{ccccc}
-\frac{2\left(m_{0}+1\right)}{z} & 1 & & & \\
-1 & -\frac{2\left(m_{0}+2\right)}{z} & 1 & & \\
& \ddots & \ddots & \ddots & \\
& & -1 & -\frac{2(K-2)}{z} & 1 \\
& & -1 & -\frac{2(K-1)}{z}
\end{array}\right)}_{:=B_{m_{0}, K}(z)}\left(\begin{array}{c}
\tilde{\omega}_{m_{0}+1} \\
\tilde{\omega}_{m_{0}+2} \\
\vdots \\
\tilde{\omega}_{K-2} \\
\tilde{\omega}_{K-1}
\end{array}\right)=\left(\begin{array}{c}
2 \xi_{m_{0}+1}+\tilde{\omega}_{m_{0}} \\
2 \xi_{m_{0}+2} \\
\vdots \\
2 \xi_{K-2} \\
2 \xi_{K-1}-\tilde{\omega}_{K}
\end{array}\right) \text {, }
$$

where $\tilde{\omega}_{m_{0}}$ is obtained from the first phase and the quantity $\tilde{\omega}_{K}$ is approximated accurately by an asymptotic expansion for $K$ large (see Appendix A). The question is how to determine the switch point $m_{0}$. The results shown in Fig. 7 imply that we should terminate the computation of the first phase before $m$ reaches $|z|$, i.e., $m_{0}<|z|$. However, if $m_{0}<|z|$ 

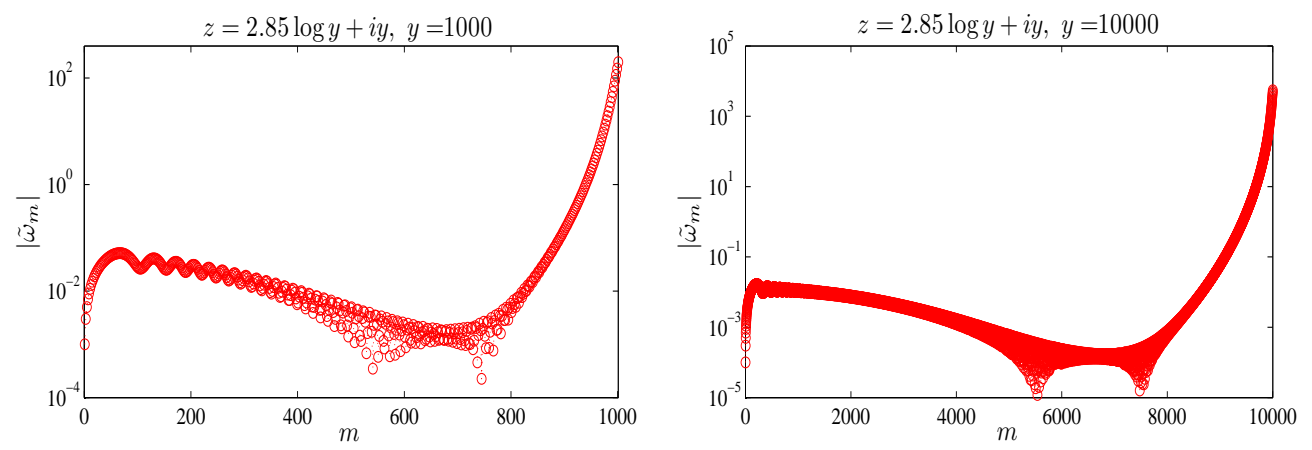

Figure 7: The quantity $\left|\tilde{\omega}_{m}\right|$ blows up before $m$ reaches $|z|$ and therefore the computation by directly forwarding the three-term recurrence (3.14) is unstable.

the tri-diagonal matrix $B_{m_{0}, K}(z)$ is no longer diagonally dominate and thus the stability analysis given in [8, Section 5] is unapplicable in this situation.

Here, we propose an idea for computing $\left\{\tilde{\omega}_{m}\right\}_{m=0}^{M}$ as follows. First, the switch point $m_{0}$ between the first and second phases is determined automatically by checking the computational error bound for the three-term recurrence (3.14). If the measured error bound reaches the prescribed tolerance $\delta$, we turn to the second phase. Then, for second phase we solve the tri-diagonal linear system (3.15) by using the Gaussian elimination, which is a fast method since the matrix $B_{m_{0}, K}(z)$ is tri-diagonal with uniform off-diagonals.

\section{- Description of the first phase computation}

For the recurrence (3.14), taking the rounding errors $\left\{\varrho_{m}\right\} \subset \mathbb{C}$ (with $\left|\varrho_{m}\right| \leq \varrho$ ) into account, we actually obtain $\left\{\tilde{\tilde{\omega}}_{j}\right\}$ and $\left\{\tilde{\omega}_{j}\right\}$ as

$$
\tilde{\omega}_{1}=\tilde{\omega}_{1}+\varrho_{1}, \quad \tilde{\omega}_{2}=\tilde{\omega}_{2}+\varrho_{2}, \quad \tilde{\tilde{\omega}}_{m+1}-\frac{2 m}{z} \tilde{\omega}_{m}-\tilde{\omega}_{m-1}=2 \xi_{m}+\varrho_{m} \quad \text { for } m \geq 3 .
$$

Denote the error by $\delta_{m}:=\tilde{\tilde{\omega}}_{m}-\tilde{\omega}_{m}$. Then, these errors satisfy

$$
\delta_{m+1}=\frac{2 m}{z} \delta_{m}+\delta_{m-1}+\varrho_{m}, \quad m=2,3, \cdots, \quad \text { with } \delta_{1}=\varrho_{1}, \quad \delta_{2}=\varrho_{2} .
$$

In practice, we can use eps (the spacing of floating point numbers) as a measure of the rounding errors $\left\{\varrho_{m}\right\}$ and this implies that the quantity $\bar{\delta}_{m}$ determined by

$$
\bar{\delta}_{m+1}=\frac{2 m}{|z|} \bar{\delta}_{m}+\bar{\delta}_{m-1}+\text { eps, } \quad m=2,3, \cdots, \quad \text { with } \bar{\delta}_{1}=\text { eps, } \bar{\delta}_{2}=\text { eps },
$$

shall be a credible prediction of the error $\left|\delta_{m}\right|$. A more sharp prediction can be obtained by simply keeping $z$ instead of $|z|$ in the above recurrence, i.e.,

$$
\bar{\delta}_{m+1}=\frac{2 m}{z} \bar{\delta}_{m}+\bar{\delta}_{m-1}+\text { eps, } \quad m=2,3, \cdots, \quad \text { with } \bar{\delta}_{1}=\text { eps, } \bar{\delta}_{2}=\text { eps } .
$$

In this case, there is however no guarantee that it exactly holds $\left|\delta_{m}\right| \leq\left|\bar{\delta}_{m}\right|$ for every $m$, but our various numerical experiments indicate that as $m$ increases $\left|\bar{\delta}_{m}\right|$ predicts $\left|\delta_{m}\right|$ very 
well. A MATLAB function FCCtw1 for computing $\left\{\tilde{\omega}_{m}\right\}_{m=3}^{m_{0}}$ in practice is given in the following (delta denotes the prescribed error tolerance $\delta$ ):

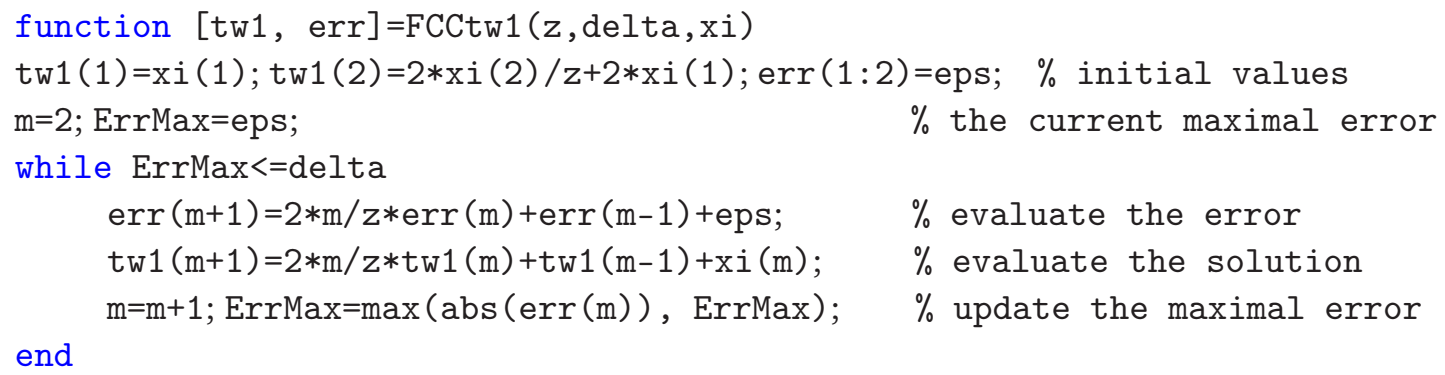

- Description of the second phase computation

When the switch point $m_{0}$ is fixed, we turn to the computation of the second phase, i.e., solving the linear system (3.15). Applying the Gaussian elimination to (3.15) gives

$$
\left\{\begin{array}{c}
b_{m_{0}+1} \tilde{\omega}_{m_{0}+1}+\tilde{\omega}_{m_{0}+2}=y_{m_{0}+1}, \\
b_{m_{0}+2} \tilde{\omega}_{m_{0}+2}+\tilde{\omega}_{m_{0}+3}=y_{m_{0}+2}, \\
\vdots \\
b_{K-1} \tilde{\omega}_{K-1}=y_{K-1},
\end{array}\right.
$$

where $\left\{b_{m}\right\}_{m=m_{0}+1}^{K-1}$ and $\left\{y_{m}\right\}_{m=m_{0}+1}^{K-1}$ are defined by

$$
\left\{\begin{array}{l}
b_{m+1}=-\frac{2(m+1)}{z}+\frac{1}{b_{m}} \text { for } m=m_{0}+1, m_{0}+2, \cdots, K-2 \text { with } b_{m_{0}+1}=-\frac{2\left(m_{0}+1\right)}{z}, \\
y_{m+1}=2 \xi_{m+1}+\frac{y_{m}}{b_{m}} \text { for } m=m_{0}+1, m_{0}+2, \cdots, K-3, \\
\text { with } y_{m_{0}+1}=2 \xi_{m_{0}+1}+\tilde{\omega}_{m_{0}} \text { and } y_{K-1}=2 \xi_{K-1}-\tilde{\omega}_{K}+\frac{y_{K-2}}{b_{K-2}} .
\end{array}\right.
$$

The left boundary value $\tilde{\omega}_{m_{0}}$ is obtained in the first phase (i.e., by directly forwarding the three-term recurrence (3.14)) and the right boundary value $\tilde{\omega}_{M}$ is approximated by an asymptotic expansion given in Appendix A. When we get $\left\{b_{m}\right\}_{m=m_{0}+1}^{K-1}$ through (3.18), then it is easy to obtain $\left\{\tilde{\omega}_{m}\right\}_{m=m_{0}+1}^{K-1}$ from (3.17) via a backward substitution.

Taking the rounding errors into account, we actually obtain these quantities as

$$
\begin{aligned}
& \left\{\begin{array}{l}
\tilde{b}_{m+1}=-\frac{2(m+1)}{z}+\frac{1}{\tilde{b}_{m}}+\varrho_{a, m} \text { for } m=m_{0}+1, \cdots, K-2, \\
\text { with } \tilde{b}_{m_{0}+1}=-\frac{2\left(m_{0}+1\right)}{z}+\varrho_{a, m_{0}},
\end{array}\right. \\
& \left\{\begin{array}{l}
\tilde{y}_{m+1}=2 \xi_{m+1}+\frac{\tilde{y}_{m}}{\tilde{b}_{m}}+\varrho_{y, m} \\
\text { for } m=m_{0}+1, m_{0}+2, \cdots, K-3, \\
\text { with } \tilde{y}_{m_{0}+1}=2 \xi_{m_{0}+1}+\tilde{\omega}_{m_{0}}+\varrho_{y, m_{0}} \\
\text { and } \tilde{y}_{K-1}=2 \xi_{K-1}-\tilde{\omega}_{K}+\frac{\tilde{y}_{K-2}}{\tilde{b}_{K-2}}+\varrho_{y, K-2},
\end{array}\right. \\
& \left\{\begin{array}{l}
\tilde{b}_{m} \tilde{\tilde{\omega}}_{m}+\tilde{\tilde{\omega}}_{m+1}=\tilde{y}_{m}+\varrho_{\tilde{\omega}, m} \text { for } m=K-2, K-3, \cdots, m_{0}+1, \\
\text { with } \tilde{\tilde{\omega}}_{K-1}=\frac{\tilde{y}_{K-1}}{\tilde{b}_{K-1}}+\varrho_{\tilde{\omega}, K-1},
\end{array}\right.
\end{aligned}
$$


where $\left\{\varrho_{a, m}, \varrho_{y, m}, \varrho_{\tilde{\omega}, m}\right\}_{m=m_{0}}^{K-2}$ denote the rounding errors and satisfy $\left|\varrho_{a, m}\right|,\left|\varrho_{y, m}\right|,\left|\varrho_{\tilde{\omega}, m}\right| \leq$ $\varrho$.

Proposition 3.1. If the quantities $m_{0}$ and $z$ satisfy

$$
\frac{-2\left(m_{0}+1\right) \Re(z)}{|z|^{2}}>\varrho,
$$

then the Gaussian elimination for solving the linear system (3.15) is stable, in the sense that

$$
\begin{aligned}
& \left|\tilde{\omega}_{m}-\tilde{\tilde{\omega}}_{m}\right| \\
\leq & \varrho \tilde{C}(\varrho)\left(1+\sum_{l=0}^{K-m-2} \frac{1}{d_{\varrho}^{l+1} m(m+1) \cdots(m+l)}\right), \quad m_{0}+1 \leq m \leq K-1,
\end{aligned}
$$

where $\tilde{C}(\varrho)$ is a bounded value (as $\varrho \rightarrow 0$ ) and $d_{\varrho}=\frac{-2 \Re(z)}{|z|^{2}}-\frac{\varrho}{m_{0}+1}$.

Proof. The proof is technical and is presented in Appendix B. ple

To test the whole procedure for computing $\left\{\tilde{\omega}_{m}\right\}_{m=3}^{M}$, we consider the following exam-

$$
\left\{\begin{array}{l}
\tilde{\omega}_{m+1}=\frac{2 m}{z} \tilde{\omega}_{m}+\tilde{\omega}_{m-1}-2\left(\frac{\sqrt{z}}{(3 \sqrt{z}+m-1)^{2}-1}+\frac{m}{\sqrt{z}(3 \sqrt{z}+m-1)}\right), \quad \Rightarrow \quad \tilde{\omega}_{m}=\frac{\sqrt{z}}{3 \sqrt{z}+m-1} . \\
\tilde{\omega}_{1}=\frac{1}{3}, \quad \tilde{\omega}_{2}=\frac{\sqrt{z}}{1+3 \sqrt{z}},
\end{array}\right.
$$

We denote the numerical solutions of (3.22) by $\left\{\tilde{\tilde{\omega}}_{m}\right\}$. Since the exact solutions $\left\{\tilde{\omega}_{m}\right\}$ are known, we can measure the error $\left|\tilde{\omega}_{m}-\tilde{\tilde{\omega}}_{m}\right|$ during the computation for each $m$. With two different tolerances $\delta$, we show in Fig. 8 the errors $\left\{\left|\tilde{\omega}_{m}-\tilde{\tilde{\omega}}_{m}\right|\right\}_{m=1}^{M}$ for two values of $z$. (The left and right subfigures correspond to $M=1200$ and $M=2800$ respectively.) For each tolerance $\delta$, the errors of the first and second phases are separated by a dashdot vertical line. For the first phase, the computational error generated by (3.16) is also shown by dotted lines. We see that the error of the first phase remains for the first few steps in the second phase and then becomes invisible as $m$ increases. Moreover, for the first phase the computational error predicts the measured error very well. We have tested many other cases of the exact solutions $\left\{\tilde{\omega}_{m}\right\}$, which, as $m$ increases, oscillate (e.g., $\tilde{\omega}_{m}=$ $\cos (1-m)+2 / z)$, approach to a constant (e.g., $\tilde{\omega}_{m}=m /(\sqrt{z}+2 m)$ ) or grow linearly $\left(\tilde{\omega}_{m}=0.5 \mathrm{~m} / z\right)$, and the plots look similar and the above conclusions also hold.

\section{Special treatment of the source term and numerical results}

For the contour integral, a serious problem is how to treat the Laplace forward transform of the source term $f(\mathbf{x}, t)$. In some cases, $\hat{f}(\mathbf{x}, s)$ also possesses singularities. If these singularities do not lie on the left of the contour $\Gamma_{\beta}$, we need to modify $\Gamma_{\beta}$ and this shall makes the contour design complicated. What makes matters worse is the cases that an 

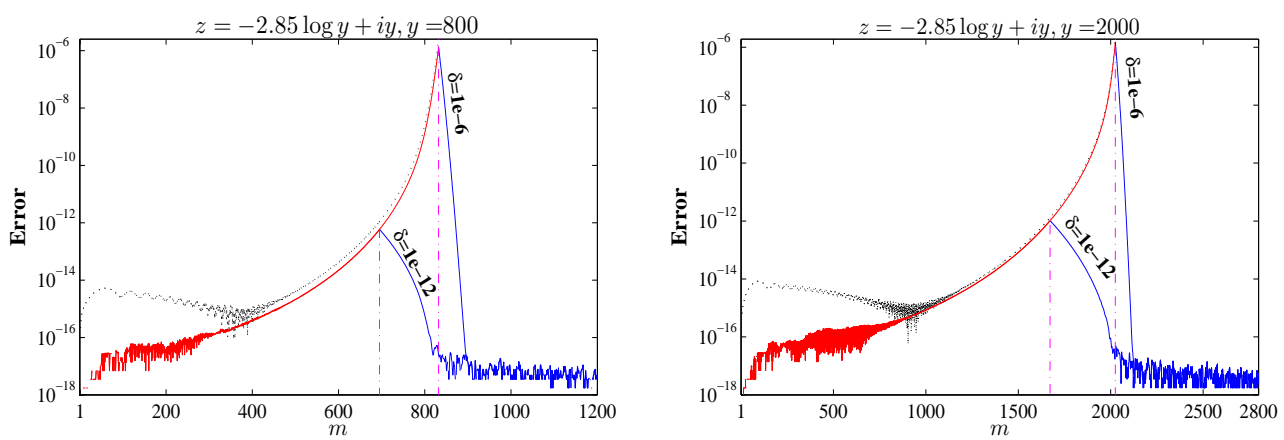

Figure 8: For the three-term recurrence (3.22) with exact solutions $\left\{\tilde{\omega}_{m}=\sqrt{z} /(3 \sqrt{z}+m-1)\right\}$, the measured error for the first and second phases, together with the computational error bound $\left\{\left|\bar{\delta}_{m}\right|\right\}$ denoted by dotted lines, where $\left\{\bar{\delta}_{m}\right\}$ are generated by (3.16).

explicit expression of $\hat{f}(\mathbf{x}, s)$ is not explicitly known. Special treatment of the source term will be the topic of this section and here we consider three cases for $\hat{f}(\mathbf{x}, s)$ and present numerical results for each case. The following setup is used throughout this section:

- The contour $\Gamma_{\beta}$ is fixed by (2.8) (or equivalently by (2.9)) with $\beta_{0}=2$ and $\beta_{1}=0.2$.

- For the truncated contour integral (2.19), the quantity $Y_{\max }$ is determined by Theorem 2.1 with a truncation error $\epsilon=1 e-8$. Then, the interval $\left[Y_{\min }, Y_{\max }\right]$ is divided into $J_{\max }+J_{\min }+2$ subintervals $\left[Y_{\min }, Y_{\max }\right]=\bigcup_{j=-J_{\min }-1}^{J_{\max }}\left[Y_{j}, Y_{j+1}\right]$ as given by (3.1), where $J_{\min }=-6$ and $J_{\max }=1+\left[\log _{B}\left(Y_{\max }-Y_{\min }\right)\right]$ with $B=8$.

- We use a uniform number of FCC nodes, say $M$ nodes, to treat all the subintervals $\left[Y_{j}, Y_{j+1}\right]$; the inner integral (3.3) and the integral over the interval $\left[0, Y_{\min }\right]$ are treated directly (without subintervals) by the FCC quadrature with $M$ nodes.

- The moments of the FCC quadrature applied to (3.3) are computed via (3.13) with $\left\{\tilde{\omega}_{m}\right\}_{m \geq 1}^{M}$ being computed by the two phase procedure introduced in Section 3.2.2. The first phase switches to the second phase when the computational error reaches a tolerance $\delta=1 e-12$.

\subsection{The case $\hat{f}(\mathbf{x}, s)$ has no singularities on the right of $\Gamma_{\beta}$}

In this case, the singularities of $\left(\left(s+a^{-s \tau}\right) I-\Delta\right)^{-1}$ are the dominate factor for the design of the contour $\Gamma_{\boldsymbol{\beta}}$. An example that satisfies this condition is the model problem (1.1), for which $\lambda=0$ the Laplace forward transform of the source term is zero.

The reader may doubt why we need to deform the Bromwich integral (2.2) to the contour integral (2.3) and why not we directly discretize the former? Indeed, the very recent work by Araújo et al. [2] implies that directly discretizing the Bromwich integral also results in efficient numerical schemes for time-dependent problems. Applying the method in [2] to 


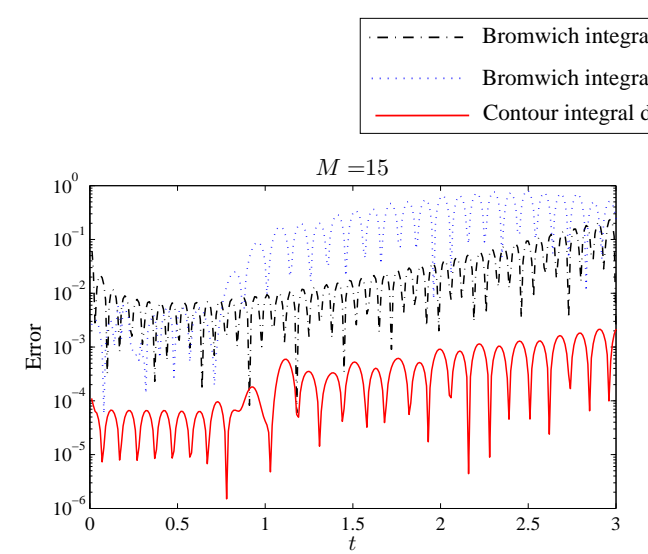

Figure 9: Accuracy comparison of the three methods for (1.1) with $\tau=0.05$. Left: $M=15$; Right: $M=30$.

our Bromwich integral (2.2) with $\lambda=0$ gives

$$
\begin{aligned}
u(t)= & \frac{1}{2 \pi i} \int_{\sigma-i \infty}^{\sigma+i \infty} e^{s t}\left(s+a e^{-s \tau}\right)^{-1} \hat{F}(s) d s \\
\approx & \frac{1}{T} e^{\sigma t}\left[\frac{\left(\sigma+a e^{-\sigma \tau}\right)^{-1} \hat{F}(\sigma)}{2}+\sum_{l=1}^{\infty} \Re\left(\left(s_{l}+a e^{-s_{l} \tau}\right)^{-1} \hat{F}\left(s_{l}\right) e^{\frac{i l \pi t}{T}}\right)\right], \\
& \text { with } s_{l}=\sigma+\frac{i l \pi}{T},
\end{aligned}
$$

which is obtained by discretizing the integral from $\sigma-i \infty$ to $\sigma+i \infty$ by the trapezoidal rule with step size $\pi / T$. In practice, we need to truncate the infinite sum to a finite one $\sum_{l=1}^{L}(\cdot)$, for which we choose $L=\left(J_{\max }+J_{\min }+2\right) M$.

Since the integrand of the Bromwich integral is highly oscillatory, we can also use the FCC quadrature as we did for the contour integral (2.3), i.e., we first truncate the infinite integral interval to a finite one and then divide the truncated interval to a few fast growing subintervals for which the FCC quadrature is used. For both methods, we choose the quantity $\sigma$ as $\sigma=x_{0}+\beta_{0}$, where $x_{0}$ denotes the maximal real part of the roots of $s+a e^{-\tau s}=0$.

For the model problem (1.1) with $\tau=0.05$, we show in Fig. 9 the measured errors of the three methods for $t \in[0,3]$. We see that, for the Bromwich integral method, the trapezoidal rule results in more accurate numerical solutions than the FCC quadrature when $M=15$ (left subfigure), while for relatively large $M$, e.g., $M=30$ (right subfigure), the FCC quadrature gives more accurate numerical solutions. For both $M=15$ and $M=30$, it is clear that the method proposed in this paper is superior than the other two methods. The results shown in Fig. 9 imply that deforming the Bromwich integral to a proper contour integral and discretizing the related integrals by the FCC quadrature really improve the accuracy of the numerical solutions.

The reader may also doubt why we need to divide the interval $\left[Y_{\min }, Y_{\max }\right]$ into fast 

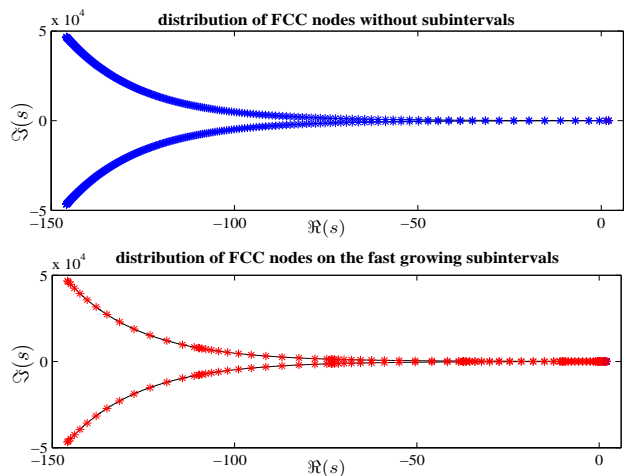

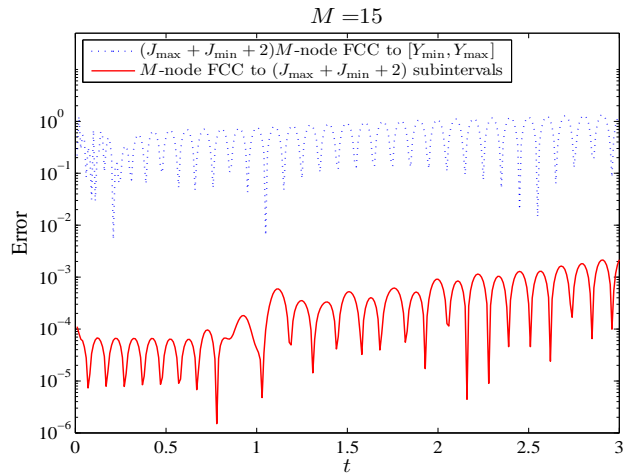

Figure 10: Left: distribution of the FCC nodes on the contour $\Gamma_{\beta}$ in two situations. Right: error of the contour integral (2.3) (with $\Gamma=\Gamma_{\beta}$ ) applied to (1.1) with problem parameters $\tau=0.05$ and $a=\pi /(2 \tau)$.

growing subintervals and why not we directly apply the FCC quadrature to $\left[Y_{\min }, Y_{\max }\right]$ ? In Fig. 10 on the left we show the distribution of the FCC quadrature nodes on the contour $\Gamma_{\beta}$ in two situations: in the top panel we show the $\left(J_{\max }+J_{\min }+2\right) M$ nodes of the FCC quadrature directly applied to $\left[Y_{\min }, Y_{\max }\right]$ and in the bottom panel we show the $M$ nodes of the FCC quadrature applied to each subinterval (there are $J_{\max }+J_{\min }+2$ subintervals in total). In the right subfigure, we show the measured error for the contour integral (2.3) with $\Gamma=\Gamma_{\boldsymbol{\beta}}$ in these two situations and this comparison implies that dividing the interval $\left[Y_{\min }, Y_{\max }\right]$ into fast growing subintervals plays an essential role to get accurate numerical solution.

\subsection{The case $\hat{f}(\mathbf{x}, s)$ has singularities on the right of $\Gamma_{\beta}$}

In this and the next subsections, we consider the following PDE with time-delay:

$$
\begin{cases}\partial_{t} u(x, t)-\partial_{x x} u(x, t)+a u(x, t-\tau)=f(x, t), & (x, t) \in(0,1) \times(0, T), \\ \partial_{x} u(0, t)=\partial_{x} u(1, t)=0, & t \in(0, T), \\ u(x, t)=x^{3}(1-x)^{3} \sin \left(\varphi t+\varphi_{0}\right), & (x, t) \in(0,1) \times[-\tau, 0],\end{cases}
$$

where $a \in \mathbb{R} \backslash\{0\}$ and the source term $f(x, t)$ is given by

$$
f(x, t)=f_{1}(x)\left[\varphi \cos \left(\varphi t+\varphi_{0}\right)+a \sin \left(\varphi(t-\tau)+\varphi_{0}\right)\right]+f_{2}(x) \sin \left(\varphi t+\varphi_{0}\right),
$$

with $f_{1}(x)=x^{3}(1-x)^{3}$ and $f_{2}(x)=6 x(x-1)\left(5 x^{2}-5 x+1\right)$. The Laplace transform of $f$ is

$$
\hat{f}(x, s)=\frac{s \hat{f}_{1}(x)+\hat{f}_{2}(x)}{s^{2}+\varphi^{2}}
$$

where

$$
\begin{aligned}
& \hat{f}_{1}(x)=\varphi \cos \left(\varphi_{0}\right) f_{1}(x)+\sin \left(\varphi_{0}\right) f_{2}(x)+a \sin \left(\varphi_{0}-\varphi \tau\right) f_{1}(x), \\
& \hat{f}_{2}(x)=\varphi \cos \left(\varphi_{0}\right) f_{2}(x)-\varphi^{2} \sin \left(\varphi_{0}\right) f_{1}(x)+a \varphi \cos \left(\varphi_{0}-\varphi \tau\right) f_{1}(x) .
\end{aligned}
$$


The exact solution $u(x, t)$ of $(4.2)$ is

$$
u(x, t)=x^{3}(1-x)^{3} \sin \left(\varphi t+\varphi_{0}\right), \quad(x, t) \in[0,1] \times[0, T] .
$$

To reduce the effect of spatial discretization on the accuracy of the numerical solutions as much as possible, we discretize the Laplacian $\partial_{x x}$ by the 4th-order compact finite-difference scheme $[10,35]$ with step-size $\Delta x=\frac{1}{100}$, which results in the following system of DDEs

$$
\begin{cases}\mathbf{u}^{\prime}(t)+A \mathbf{u}(t)+a \mathbf{u}(t-\tau)=\mathbf{f}(t), & t \in(0, T), \\ \mathbf{u}(t)=\mathbf{u}_{0}(t), & t \in[-\tau, 0]\end{cases}
$$

where $A=A_{1}^{-1} A_{2}$ and $A_{1}$ and $A_{2}$ are defined by

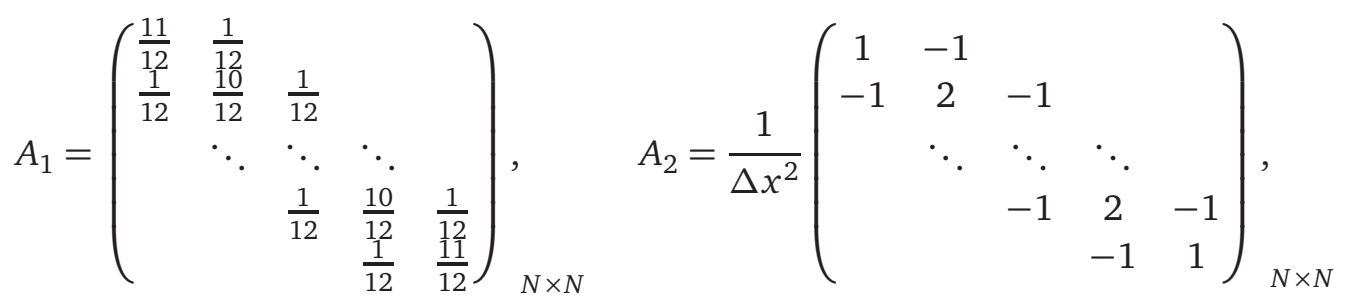

$$
\begin{aligned}
& \mathbf{f}(t)=\left(\begin{array}{cc}
f\left(x_{1}, t\right) & \\
& f\left(x_{2}, t\right) \\
\vdots & \\
f\left(x_{N}, t\right) &
\end{array}\right), \quad \mathbf{u}_{0}(t)=\left(\begin{array}{c}
u_{0}\left(x_{1}, t\right) \\
u_{0}\left(x_{2}, t\right) \\
\vdots \\
u_{0}\left(x_{N}, t\right)
\end{array}\right), \\
& N=\frac{1}{\Delta x}-1=99, \quad \quad x_{j}=j \Delta x(j=1,2, \cdots, N) .
\end{aligned}
$$

At the semi-discrete level, the contour integral (1.3) with $\Gamma=\Gamma_{\beta}$ becomes

$$
\begin{aligned}
& \mathbf{u}(t)=\frac{1}{2 \pi i} \int_{\Gamma_{\boldsymbol{\beta}}} e^{t s}\left[\left(a e^{-s \tau}+s\right) I+A\right]^{-1} \hat{F}(s) d s \\
& \quad \text { with } \hat{F}(s)=\hat{\mathbf{f}}(s)+u_{0}(0)-a \int_{0}^{\tau} e^{-s r} \mathbf{u}_{0}(r-\tau) d r .
\end{aligned}
$$

Apparently, besides the singularities of $\left[\left(a e^{-s \tau}+s\right) I+A\right]^{-1}$ the function $\hat{\mathbf{f}}(s)$ also contributes two singularities $\pm|\varphi| i$ to this contour integral; see Fig. 9 on the left for an illustration. In this case, we need to modify $\Gamma_{\boldsymbol{\beta}}$ to keep all the singularities on the left of $\Gamma_{\boldsymbol{\beta}}$. Here, we propose a method to treat the singularities of $\left[\left(a e^{-s \tau}+s\right) I+A\right]^{-1}$ and $\hat{\mathbf{f}}(s)$ separately and by using this method we do not need to modify the contour $\Gamma_{\boldsymbol{\beta}}$.

Our idea is motivated by the method proposed by Lee and Sheen $[18,19]$ and is introduced as follows. Denote by $\left\{s_{l}\right\}_{l=1}^{L}$ the singularities of $\hat{\mathbf{f}}(s)$ that lie on the right of $\Gamma_{\boldsymbol{\beta}}$ 

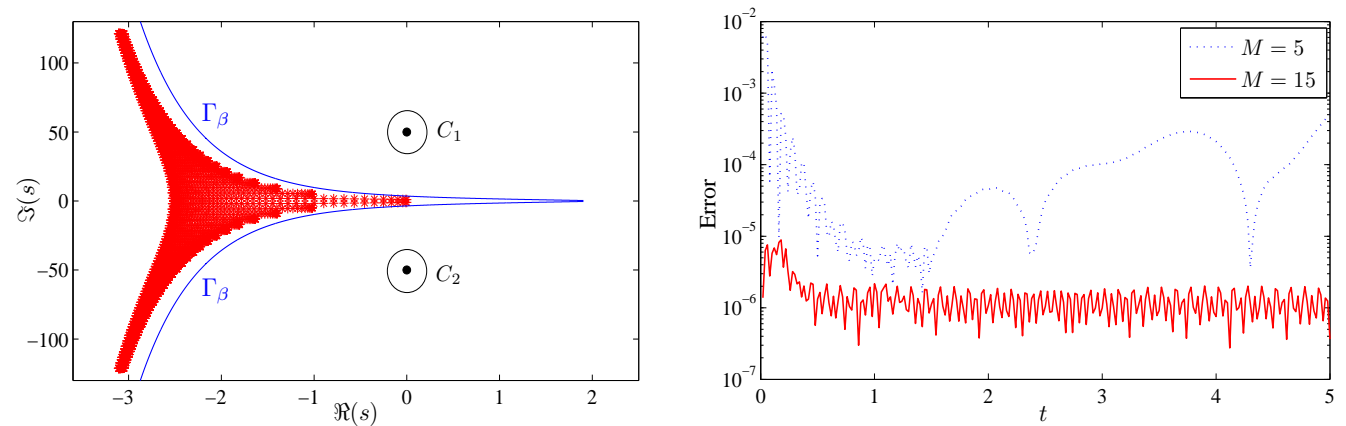

Figure 11: Left: the distribution of the singularities of $\left[\left(a e^{-s \tau}+s\right) I+A\right]^{-1} \hat{\mathrm{f}}(s)$, where the singularities of $\hat{\mathrm{f}}(s)$ lie on the right of $\Gamma_{\beta}$. Right: measured error of the method (4.9) for two values of $M$, the number of nodes for the FCC quadrature. Here, we choose $a=1, \varphi=50, \varphi_{0}=-1$ and $\tau=0.5 \pi$ for the problem parameters.

and let $C_{l}$ be the circle that centered at $s_{l}$ in the complex plane (see Fig. 11 on the left for illustration). Then, by using the Cauchy-Goursat theorem [1] we have

$$
\begin{aligned}
\mathbf{u}(t)=\frac{1}{2 \pi i} \int_{\Gamma_{\boldsymbol{\beta}}} e^{t s}\left[\left(a e^{-s \tau}\right.\right. & +s) I+A]^{-1} \hat{\mathbf{f}}(s) d s \\
& +\frac{1}{2 \pi i} \sum_{l=1}^{L} \oint_{C_{l}} e^{t s}\left[\left(a e^{-s \tau}+s\right) I+A\right]^{-1} \hat{\mathbf{f}}(s) d s,
\end{aligned}
$$

where the integral along $C_{l}$ takes the counterclockwise orientation. Then, we discretize the integral along $\Gamma_{\boldsymbol{\beta}}$ and $\left\{C_{l}\right\}$ by the FCC quadrature and the Trapezoidal rule, respectively. This method is different the one used in $[18,19]$, there the authors first fixed a semicircle with sufficiently large diameter to enclose the most significant singularities and choose small circles to surround each of these singularities; then all the integrals along the small circles are approximated by the Trapezoidal rule. Clearly, the method in $[18,19]$ is superior when the number of singularities is small and it is easy to compute the singularities accurately.

To discretize the integrals along the small circles $\left\{C_{l}\right\}$, we first parameterize $C_{l}$ as $s=\tilde{c}_{l}(\zeta):=s_{l}+\tilde{\rho} e^{i \zeta}$ and then apply the Trapezoidal rule to the resulted integral:

$$
\begin{aligned}
& \frac{1}{2 \pi i} \sum_{l=1}^{L} \oint_{C_{l}} e^{t s}\left[\left(a e^{-s \tau}+s\right) I+A\right]^{-1} \hat{\mathbf{f}}(s) d s \\
\approx & \frac{1}{2 \pi i} \sum_{l=1}^{L}\left(\frac{2 \pi}{N_{c}} \sum_{j=0}^{N_{c}-1} e^{t \tilde{c}_{l}\left(\zeta_{l, j}\right)}\left[\left(a e^{-\tilde{c}_{l}\left(\zeta_{l, j}\right) \tau}+\tilde{c}_{l}\left(\zeta_{l, j}\right)\right) I+A\right]^{-1} \hat{\mathbf{f}}\left(\tilde{c}_{l}\left(\zeta_{l, j}\right)\right) \tilde{c}_{l}^{\prime}\left(\zeta_{l, j}\right)\right) \\
= & \sum_{l=1}^{L}\left(\frac{1}{N_{c}} \sum_{j=0}^{N_{c}-1} e^{t \tilde{c}_{l}\left(\zeta_{l, j}\right)}\left[\left(a e^{-\tilde{c}_{l}\left(\zeta_{l, j}\right) \tau}+\tilde{c}_{l}\left(\zeta_{l, j}\right)\right) I+A\right]^{-1} \hat{\mathbf{f}}\left(\tilde{c}_{l}\left(\zeta_{l, j}\right)\right)\left(\tilde{\rho} e^{\left.i \zeta_{l, j}\right)}\right)\right.
\end{aligned}
$$


where $\zeta_{l, j}=2 j \pi / N_{c} \in[0,2 \pi]$. The superscript is $N_{c}-1$ instead of $N_{c}$ because

$$
\begin{aligned}
& e^{t \tilde{c}_{l}(0)}\left[\left(a e^{-\tilde{c}_{l}(0) \tau}+\tilde{c}_{l}(0)\right) I+A\right]^{-1} \hat{\mathbf{f}}\left(\tilde{c}_{l}(0)\right) \tilde{c}_{l}^{\prime}(0) \\
= & e^{t \tilde{c}_{l}(2 \pi)}\left[\left(a e^{-\tilde{c}_{l}(2 \pi) \tau}+\tilde{c}_{l}(2 \pi)\right) I+A\right]^{-1} \hat{\mathbf{f}}\left(\tilde{c}_{l}(2 \pi)\right) \tilde{c}_{l}^{\prime}(2 \pi) .
\end{aligned}
$$

Here, all the small circles are discretized by $N_{c}$ nodes (of course we can use different number of nodes for different circle). Let

$$
\mathbf{F}(s)=\left[\left(a e^{-s \tau}+s\right) I+A\right]^{-1} \hat{F}(s) .
$$

Then, from [18, Theorem 2.1] we have

$$
\left|\frac{1}{2 \pi i} \sum_{l=1}^{L} \oint_{C_{l}} e^{t s} \mathbf{F}(s) d s-\sum_{l=1}^{L}\left(\frac{1}{N_{c}} \sum_{j=0}^{N_{c}-1} \mathbf{F}\left(\tilde{c}_{l}\left(\zeta_{l, j}\right)\right)\left(\tilde{\rho} e^{i \zeta_{l, j}}\right)\right)\right|=\mathscr{O}\left(\tilde{\rho}^{N_{c}}\right) .
$$

Therefore, this method possesses geometrical convergence rate as $N_{c}$ increases. The radius $\tilde{\rho}$ plays an important role, for which we use the one given in [18, Section 2.2]:

$$
\tilde{\rho}=\operatorname{eps}^{\frac{1}{N_{c}+1}} \text {. }
$$

For numerical experiments, we choose $N_{c}=10$ and $\left(a, \tau, \varphi, \varphi_{0}\right)=(1,0.5 \pi, 50,2)$. Then, for two values of $M$, the number of nodes of the FCC quadrature used to discretize the integral along the contour $\Gamma_{\beta}$, we show in Fig. 11 on the right the error between the numerical and exact solutions for $t \in[0,5]$. With the radius $\tilde{\rho}$ given by (4.12), the error arising from discretizing the integrals along the small circles $\left\{C_{l}\right\}$ in (4.9) is negligible and therefore the error arising from discretizing the contour integrals along $\Gamma_{\beta}$ is dominate. This confirms the numerical results very well, since in Fig. 11 on the right we see that increasing $M$ really reduces the measured error.

\subsection{The case $\hat{f}(\mathbf{x}, s)$ is not known explicitly}

At the end of this section, we propose an idea for the contour integral (1.3) with $\Gamma=\Gamma_{\boldsymbol{\beta}}$ that avoids direct use of $\hat{f}(\mathbf{x}, s)$. Let

$$
\begin{aligned}
& \hat{F}_{0}(\mathbf{x}, s)=u_{0}(\mathbf{x}, 0)-a \int_{0}^{\tau} e^{-s r} u_{0}(\mathbf{x}, r-\tau) d r, \hat{\mathscr{K}}(s)=\left(\left(s+a e^{-\tau s}\right) I-\Delta\right)^{-1}, \\
& \tilde{u}(\mathbf{x}, t)=\frac{1}{2 \pi i} \int_{\Gamma_{\beta}} e^{s t} \widehat{\mathscr{K}}(s) \hat{f}(\mathbf{x}, s) d s, \\
& \mathscr{K}(t)=\mathscr{L}^{-1}(\hat{\mathscr{K}})(t):=\frac{1}{2 \pi i} \int_{\Gamma_{\beta}} e^{s t} \hat{\mathscr{K}}(s) d s .
\end{aligned}
$$

Then, we split the contour integral (1.3) with $\Gamma=\Gamma_{\beta}$ into two parts:

$$
u(\mathbf{x}, t)=\frac{1}{2 \pi i} \int_{\Gamma_{\beta}} e^{s t}\left(\left(s+a e^{-\tau s}\right) I-\Delta\right)^{-1} \hat{F}_{0}(\mathbf{x}, s) d s+\tilde{u}(\mathbf{x}, t) .
$$


For the first part, the function $\hat{F}_{0}(\mathbf{x}, s)$ is analytic on the whole complex plane and therefore the results in Sections 2 and 3 are directly applicable. For $\tilde{u}(\mathbf{x}, t)$, by using the Duhamel formula [1] we get

$$
\tilde{u}(\mathbf{x}, t)=\int_{0}^{t} \mathscr{K}(t-r) f(\mathbf{x}, r) d r=\int_{0}^{t}\left[\frac{1}{2 \pi i} \int_{\Gamma_{\beta}} e^{s(t-r)} \hat{\mathscr{K}}(s) d s\right] f(\mathbf{x}, r) d r .
$$

Thus,

$$
\tilde{u}(\mathbf{x}, t)=\frac{1}{2 \pi i} \int_{\Gamma_{\boldsymbol{\beta}}}\left(\left(s+a e^{-s \tau}\right) I-\Delta\right)^{-1} \int_{0}^{t} e^{s(t-r)} f(\mathbf{x}, r) d r,
$$

where $e^{s t}$ is included in the inner integrand to avoid floating overflow. Finally, substituting (4.15) into (4.14) gives a new representation of $u(\mathbf{x}, t)$ :

$$
u(\mathbf{x}, t)=\frac{1}{2 \pi i} \int_{\Gamma_{\boldsymbol{\beta}}} e^{s t} \hat{\mathscr{K}}(s) \hat{F}_{0}(\mathbf{x}, s) d s+\frac{1}{2 \pi i} \int_{\Gamma_{\boldsymbol{\beta}}} \hat{\mathscr{K}}(s) \int_{0}^{t} e^{s(t-r)} f(\mathbf{x}, r) d r
$$

where $\hat{\mathscr{K}}(s)$ is given by (4.13). After spatial discretization, e.g., by the 4th-order compact finite-difference formula described in Section 4.2, we have

$$
\begin{aligned}
\mathbf{u}(t)= & \frac{1}{2 \pi i} \int_{\Gamma_{\boldsymbol{\beta}}} e^{s t}\left[\left(a+s^{-\tau s}\right) I+A\right]^{-1} \hat{\mathbf{f}}_{0}(s) d s \\
& +\frac{1}{2 \pi i} \int_{\Gamma_{\boldsymbol{\beta}}}\left[\left(a+s^{-\tau s}\right) I+A\right]^{-1} \int_{0}^{t} e^{s(t-r)} \mathbf{f}(r) d r
\end{aligned}
$$

where $\hat{\mathbf{f}}_{0}(s)=\left(\hat{F}\left(x_{1}, s\right), \cdots, \hat{F}\left(x_{N}, s\right)\right)^{\top}$. Now, in (4.16) we do not need $\hat{f}(\mathbf{x}, s)$.

For the inner integral $\int_{0}^{t} e^{s(t-r)} \mathbf{f}(r) d r$ in (4.16), we also use the FCC quadrature with $M$ nodes to derive an approximation. For two values of $M, M=5$ and $M=15$, we show in Fig. 12 the measured error between the numerical and exact solutions for $t \in[0,5]$. We use two methods to compute the numerical solution: the one based on the CauchyGoursat theorem as described by (4.9) and the one based on the Duhamel formula as given by (4.16). The errors correspond to these two methods are indicated by dotted and solid lines in each panel of Fig. 12, respectively. The results shown in this figure imply that the modified contour integral (4.16) gives equally accurate numerical solution.

\section{Conclusions}

We proposed a method for solving a class of representative time-delay differential equations, which is meshless and highly parallelizable. The method consists of two components, representation of the exact solution by (truncated) contour integral and approximation of the related highly-oscillatory integrals by the Filon-Clenshaw-Curtis quadrature. The contour design is based on a careful justification of the roots of $s+a e^{-s \tau}+\lambda=0$ in the 

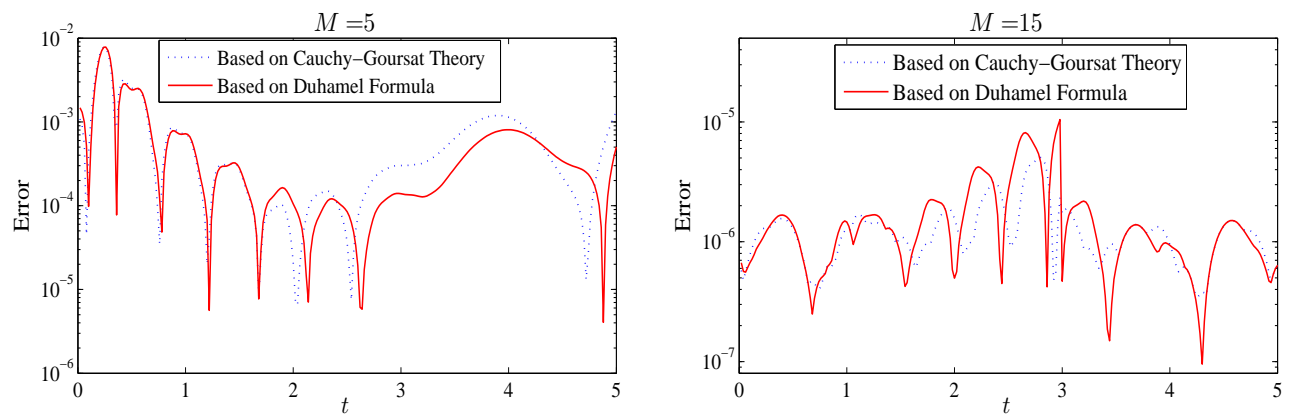

Figure 12: The measured error of the contour integral for the time-delay PDE (4.2) with two different treatments for the source term $f(x, t)$ : by the dotted line we treat the singularities of $\hat{f}(x, s)$ by the Cauchy-Goursat theorem as described by (4.9) and by the solid line we treat the source term by the Duhamel formula as given by (4.16). Here, we choose $a=1, \varphi=7, \varphi_{0}=2$ and $\tau=0.5 \pi$ for the problem parameters.

complex plane, where $\lambda \geq 0$ denotes an arbitrary eigenvalue of the negative Laplacian. The Filon-Clenshaw-Curtis quadrature is applied to two types of integrals: the one contains an exponential term $e^{i k y}$ (with $k \in \mathbb{R}$ ) as a part of the integrand and the other one contains $e^{i z y}$ (with $z \in \mathbb{C}$ ). The former was already extensively studied in the literature and in this paper we presented a detailed study for the latter. To successfully get an accurate numerical solution, the contour design must take into account the singularities of $\hat{f}$ (the Laplace forward transform of the source term), too. Two ideas were proposed in Sections 4.2 and 4.3 to treat the function $\hat{f}$ and numerical results imply that the modified contour integral based on these two ideas works equally well.

\section{Appendix A: Asymptotic expansion of $\tilde{\omega}_{K}$ for $K$ large}

To supply the right boundary value for the computation of the second phase (i.e., solving the tri-diagonal linear system (3.15)), we need to fix $\tilde{\omega}_{K}$. This quantity can be determined by the following asymptotic expansion with high accuracy.

Proposition A.1. Let $K \geq\left(2|z|^{2}\right) /(|\mathfrak{I}(z)|)$ be an integer and

$$
\left\{\begin{array}{l}
p_{0}(\phi)=\frac{1}{i K+z \sin (\phi)}, \\
p_{l}(\phi)=p_{0}(\phi) \frac{d p_{l-1}(\phi)}{d \phi}, \quad l=1,2, \cdots .
\end{array}\right.
$$

Then, it holds that

$$
\tilde{\omega}_{K}(z):=\frac{1}{K} \int_{-1}^{1} T_{K}^{\prime}(y) e^{z(1-y)} d y=i\left(\sum_{l=0}^{2 \tilde{N}+2}(-1)^{l} p_{l}(0)\left[1+(-1)^{l+1} e^{2 z}\right]\right)+R_{\tilde{N}}
$$


where the truncation term $R_{\tilde{N}}$ satisfies

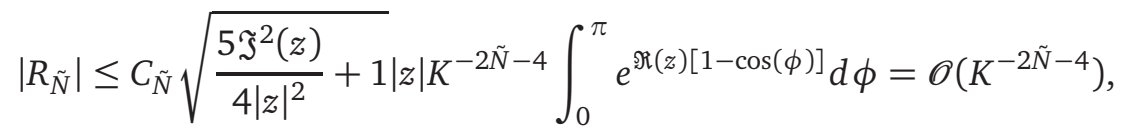

with $C_{\tilde{N}}$ being a constant independent of $K$ and $z$.

Proof. The proof of this proposition is essentially the same as that of [8, Thm. 3.1].

For reader's convenience, we give the fist 9 coefficients in the asymptotic formula (A.1):

$$
\begin{aligned}
& p_{0}(0)=\frac{1}{i K}, \quad p_{1}(0)=-\frac{z}{(i K)^{3}}, \quad p_{2}(0)=\frac{3 z^{2}}{(i K)^{5}}, \quad p_{3}(0)=z \frac{(i K)^{2}-15 z^{2}}{(i K)^{7}}, \\
& p_{4}(0)=15 z^{2} \frac{7 z^{2}-(i K)^{2}}{(i K)^{9}}, \quad p_{5}(0)=-\frac{945 z^{4}-210 z^{2}(i K)^{2}+(i K)^{4}}{(i K)^{11}}, \\
& p_{6}(0)=z^{2} \frac{-3150 z^{2}(i K)^{2}+63(i K)^{4}+10395 z^{4}}{(i K)^{13}}, \\
& p_{7}(0)=z \frac{(i K)^{6}-2205(i K)^{4} z^{2}+51975(i K)^{2} z^{4}-135135 z^{6}}{(i K)^{15}}, \\
& p_{8}(0)=15 z^{2} \frac{135135 z^{6}-63063 z^{4}(i K)^{2}+4389 z^{2}(i K)^{4}-17(i K)^{6}}{(i K)^{17}} .
\end{aligned}
$$

These coefficients are obtained by using the symbolic toolbox in Matlab.

\section{Appendix B: Proof of proposition 3.1}

Proof. Condition (3.20) implies $\Re(z)<0$. For $m \geq m_{0}+1$, we claim

$$
\left|\tilde{b}_{m}\right| \geq \Re\left(\tilde{b}_{m}\right) \geq d_{\varrho} m, \quad \text { with } d_{\varrho}=\frac{-2 \Re(z)}{|z|^{2}}-\frac{\varrho}{m_{0}+1} .
$$

Under condition (3.20), we have $d_{\varrho}>0$ and $\frac{-2 m \Re(z)}{|z|^{2}}-\varrho \geq d_{\varrho} m$ for $m \geq m_{0}+1$. Since

$$
\Re\left(\tilde{b}_{m_{0}+1}\right) \geq-\frac{2\left(m_{0}+1\right) \Re(z)}{|z|^{2}}-\varrho,
$$

we know that (B.3) holds for $m=m_{0}+1$. Assume that (B.3) holds for some $m>m_{0}+1$. Then,

$$
\Re\left(\tilde{b}_{m+1}\right)=-\frac{2 \Re(z)(m+1)}{|z|^{2}}+\frac{\Re\left(\tilde{b}_{m}\right)}{\left|\tilde{b}_{m}\right|^{2}}+\Re\left(\varrho_{a, m}\right) \geq-\frac{2 \Re(z)(m+1)}{|z|^{2}}+\frac{\Re\left(\tilde{b}_{m}\right)}{\left|\tilde{b}_{m}\right|^{2}}-\varrho .
$$

Since $\Re\left(\tilde{b}_{m}\right) \geq d_{\varrho} m>0$, we can simply omit the term $\Re\left(\tilde{b}_{m}\right) /\left|\tilde{b}_{m-1}\right|^{2}$ and get

$$
\Re\left(\tilde{b}_{m+1}\right) \geq-\frac{2 \Re(z)(m+1)}{|z|^{2}}-\varrho \geq d_{\varrho}(m+1) .
$$


Hence, (B.3) also holds for $m+1$. By letting $\varrho=0$, it is easy to deduce that the quantities $\left\{b_{m}\right\}_{m=m_{0}+1}^{K-1}$ of (3.18) satisfy

$$
\left|b_{m}\right| \geq d_{0} m \quad \text { for } m \geq m_{0}+1 .
$$

Let

$$
\begin{aligned}
& C_{\varrho}=\max \left\{\left|\tilde{\omega}_{m_{0}}\right|,\left|\tilde{\omega}_{K}\right|\right\}+2 \max _{m \geq m_{0}}\left|\xi_{m}\right|+\varrho, \\
& \psi_{r, \varrho}=1+\max _{m \geq m_{0}+1} \frac{1}{d_{\varrho}^{r l}[(m-1)(m-2) \cdots(m-l)]^{r}} .
\end{aligned}
$$

Then, by using (B.3) and (B.4) we have (by a method of induction)

$$
\begin{aligned}
& \left|\tilde{y}_{m}\right| \leq C_{\varrho}\left(1+\sum_{l=1}^{m-m_{0}-1} \frac{1}{d_{\varrho}^{l}(m-1)(m-2) \cdots(m-l)}\right) \leq C_{\varrho} \psi_{1, \varrho}, \quad \forall m \geq m_{0}+2, \\
& \left|y_{m}\right| \leq C_{0}\left(1+\sum_{l=1}^{m-m_{0}-1} \frac{1}{d_{0}^{l}(m-1)(m-2) \cdots(m-l)}\right) \leq C_{0} \psi_{1,0}, \quad \forall m \geq m_{0}+2 .
\end{aligned}
$$

From (3.18)-(3.19a) and (B.3)-(B.4), we have

$$
\left|\tilde{b}_{m+1}-b_{m+1}\right| \leq\left|\frac{\tilde{b}_{m}-b_{m}}{\tilde{b}_{m} b_{m}}\right|+\varrho \leq \frac{\left|\tilde{b}_{m}-b_{m}\right|}{d_{0} d_{\varrho} m^{2}}+\varrho,
$$

which, together with $\left|\tilde{b}_{m_{0}+1}-b_{m_{0}+1}\right| \leq \varrho$ and a method of induction, gives

$$
\begin{aligned}
\left|\tilde{b}_{m}-b_{m}\right| & \leq \varrho\left(1+\sum_{p=1}^{m-m_{0}-1} \frac{1}{\left(d_{0} d_{\varrho}\right)^{p}[(m-1)(m-2) \cdots(m-p)]^{2}}\right) \\
& \leq \varrho \psi_{2, \varrho}, \quad \forall m \geq m_{0}+1,
\end{aligned}
$$

where we have used $d_{0}>d_{\varrho}>0$. We next estimate $\left|\tilde{y}_{m+1}-y_{m+1}\right|$. We have

$$
\begin{aligned}
\left|\tilde{y}_{m+1}-y_{m+1}\right| & \leq \frac{\left|y_{m}\right|\left|\tilde{b}_{m}-b_{m}\right|+\left|a_{m}\right|\left|\tilde{y}_{m}-y_{m}\right|}{\left|\tilde{b}_{m} b_{m}\right|}+\varrho \\
& \leq \frac{\left|y_{m}\right|\left|\tilde{b}_{m}-b_{m}\right|}{d_{0} d_{\varrho} m^{2}}+\frac{1}{d_{\varrho} m}\left|\tilde{y}_{m}-y_{m}\right|+\varrho .
\end{aligned}
$$

From above estimates for $\left|y_{m}\right|$ and $\left|\tilde{b}_{m}-b_{m}\right|$, it is obvious that

$$
\frac{\left|y_{m}\right|\left|\tilde{b}_{m}-b_{m}\right|}{d_{0} d_{\varrho} m^{2}} \leq \varrho C_{2}\left(\forall m \geq m_{0}+1\right), \quad \text { with } C_{2}=\frac{C_{0} \psi_{1,0} \psi_{2, \varrho}}{d_{0} d_{\varrho}\left(m_{0}+1\right)^{2}} .
$$


By using $\left|\tilde{y}_{m_{0}+1}-y_{m_{0}+1}\right| \leq \varrho$ and by substituting (B.7) into (B.6), it holds that

$$
\begin{aligned}
\left|\tilde{y}_{m}-y_{m}\right| & \leq\left(C_{2}+1\right)\left(1+\sum_{p=1}^{m-m_{0}-1} \frac{1}{d_{\varrho}^{p}(m-1)(m-2) \cdots(m-p)}\right) \varrho \\
& \leq \varrho\left(C_{2}+1\right) \psi_{1, \varrho} .
\end{aligned}
$$

Now, we are ready to prove (3.21). Since

$$
\tilde{y}_{m+1}-y_{m+1}=\frac{\tilde{y}_{m}}{\tilde{b}_{m}}-\frac{y_{m}}{b_{m}}+\varrho_{y, m}\left(\Rightarrow\left|\frac{\tilde{y}_{m}}{\tilde{b}_{m}}-\frac{y_{m}}{b_{m}}\right| \leq\left|\tilde{y}_{m+1}-y_{m+1}\right|+\varrho\right),
$$

it follows by using (B.8) that

$$
\begin{aligned}
& \left|\frac{\tilde{y}_{K-1}}{\tilde{b}_{K-1}}-\frac{y_{K-1}}{b_{K-1}}\right| \leq\left[\left(C_{2}+1\right) \psi_{1, \varrho}+1\right] \varrho \\
\Rightarrow & \left|\tilde{\tilde{\omega}}_{K-1}-\tilde{\omega}_{K-1}\right| \leq\left[\left(C_{2}+1\right) \psi_{1, \varrho}+2\right] \varrho .
\end{aligned}
$$

From (3.17) and (3.19c), we have

$$
\tilde{b}_{m}\left(\tilde{\tilde{\omega}}_{m}-\tilde{\omega}_{m}\right)+\left(\tilde{b}_{m}-b_{m}\right) \tilde{\omega}_{m}=\left(\tilde{y}_{m}-y_{m}\right)+\left(\tilde{\tilde{\omega}}_{m+1}-\tilde{\omega}_{m+1}\right)+\varrho_{\tilde{\omega}, m},
$$

which gives

$$
\begin{aligned}
& \left|\tilde{\tilde{\omega}}_{m}-\tilde{\omega}_{m}\right| \\
\leq & \frac{1}{\left|\tilde{b}_{m}\right|}\left(\left|\tilde{b}_{m}-b_{m}\right|\left|\tilde{\omega}_{m}\right|+\left|\tilde{y}_{m}-y_{m}\right|+\varrho\right)+\frac{1}{\left|\tilde{b}_{m}\right|}\left|\tilde{\tilde{\omega}}_{m+1}-\tilde{\omega}_{m+1}\right|, \quad \forall m \leq K-2 .
\end{aligned}
$$

It is easy to know that $\left|\tilde{\omega}_{m}\right|$ is bounded for all $m \geq 1$. This, together with (B.5) and (B.8), gives

$$
\begin{aligned}
\max _{m \geq m_{0}+1} & \frac{1}{\left|\tilde{b}_{m}\right|}\left(\left|\tilde{b}_{m}-b_{m}\right|\left|\tilde{\omega}_{m}\right|+\left|\tilde{y}_{m}-y_{m}\right|+\varrho\right) \leq \varrho C_{3}, \\
& \text { with } C_{3}=\frac{\psi_{2, \varrho} \max _{m \geq m_{0}+1}\left|\tilde{\omega}_{m}\right|+\left(C_{2}+1\right) \psi_{1, \varrho}+1}{d_{\varrho}\left(m_{0}+1\right)} .
\end{aligned}
$$

Let $\tilde{C}(\varrho)=\max \left\{C_{3},\left(C_{2}+1\right) \psi_{1, \varrho}+2\right\}$. Then, from (B.9) and (B.10) it holds that

$$
\left\{\begin{array}{l}
\left|\tilde{\omega}_{m}-\tilde{\omega}_{m}\right| \leq \varrho \tilde{C}(\varrho)+\frac{1}{d_{\varrho} m}\left|\tilde{\omega}_{m+1}-\tilde{\omega}_{m+1}\right|, \quad \forall m \leq K-2, \\
\left|\tilde{\tilde{\omega}}_{K-1}-\tilde{\omega}_{K-1}\right| \leq \varrho \tilde{C}(\varrho),
\end{array}\right.
$$

which, together with a method of induction, leads to (3.21).

Since $d_{\varrho}$ is a decreasing function of $\varrho, \psi_{r, \varrho}$ is an increasing function of $\varrho$, which implies that $C_{2}$ and $C_{3}$ are increasing functions of $\varrho$. Hence, $\lim _{\varrho \rightarrow 0} \tilde{C}(\varrho)$ is a finite value. 
Acknowledgments The first author was supported by NSFC (No. 11771313, No. 615730 10) and the Project of China Postdoctoral Science Foundation (No. 2015M580777, No. 201 6T90841). The second author was supported by NSFC (No. 11771163).

\section{References}

[1] L. V. Ahlfors, Complex Analysis, 3rd ed, McGraw-Hill, New Yourk, 1978.

[2] A. Araújo, A. K. Das, C. Neves and E. Sousa, Numerical solution for a non-Fickian diffusion in a periodic potential, Commun. Comput. Phys., 13 (2013), pp. 502-525.

[3] A. Bellen and M. Zennaro, Numerical Methods for Delay Differential Equations, Oxford university press, 2013.

[4] C. T. H. BAKer, C. A. H. Paul And D. R. Wille, Issues in the numerical solution of evolutionary delay differential equations, Adv. Comp. Math., 3 (1995), pp. 171-196.

[5] C. BRIAT, Linear Parameter-Varying and Time-Delay Systems: Analysis, Observation, Filtering and Control, Springer-Verlag, Heidelberg, 2015.

[6] P. H. Cocquet and M. J. Gander, How large a shift is needed in the shifted Helholtz preconditioner for its effective inversion by multigrid? SIAM J. Sci. Comput., 39 (2017), pp. A438-A478.

[7] G. DAhlquist And Å. BJöRCK, Numerical Methods in Scientific Computing, vol. I, SIAM, Philadelphia 55, 2007.

[8] V. Domínguez, I. G. Graham and V. P. Smyshlyaev, Stability and error estimates for FilonClenshaw-Curtis rules for highly oscillatory integrals, IMA J. Numer. Anal., 31 (2011), pp. 12 53-1280.

[9] V. Domínguez, I. G. Graham and T. Kim, Filon-Clenshaw-Curtis rules for highly oscillatory integrals with algebraic singularities and stationary points, SIAM J. Numer. Anal., 51 (2013), pp. 1542-1566.

[10] G. H. GaO And H. W. Sun, Three-point combined compact alternating direction implicit difference schemes for two-dimensional time-fractional advection-diffusion equations, Commun. Comput. Phys., 17 (2015), pp. 487-509.

[11] M. J. Gander, M. Al-Khaleel And A. E. Ruehli, Waveform relaxation technique for longitudinal partitioning of transmission lines, Electrical Performance of Electronic Packaging, 2006, pp. 207-210.

[12] D. Huybrechs and S. Vandewalle, On the evaluation of highly oscillatory integrals by analytic continuation, SIAM J. Numer. Anal., 44 (2006), pp. 1026-1048.

[13] C. M. HuAng AND S. VANDEwalle, Unconditionally stable difference methods for delay partial differential equations, Numer. Math., 122 (2012), pp. 579-601.

[14] C. M. Huang, Delay-dependent stability of high order Runge-Kutta methods, Numer. Math., 111 (2009), pp. 377-0387.

[15] A. IsERles AND S. P. NøRSETt, Efficient quadrature of highly oscillatory integrals using derivatives, Proc. Royal Soc. A, 461 (2005), pp. 1383-1399.

[16] A. IsERLES, On the numerical quadrature of highly-oscillating integrals I: Fourier transforms, IMA J. Numer. Anal., 24 (2004), pp. 365-391.

[17] Y. L. JIANG, On time-domain simulation of lossless transmission lines with nonlinear terminations, SIAM J. Numer. Anal., 42 (2004), pp. 1018-1031.

[18] J. LeE And D. Sheen, An accurate numerical inversion of Laplace transforms based on the location of their poles, Comput. Math. Appl., 48 (2004), pp. 1415-1423.

[19] J. LEE AND D. SHEEN, A parallel method for backward parabolic problems based on the Laplace transformation, SIAM J. Numer. Anal., 44 (2006), pp. 1466-1486. 
[20] C. Lubich And A. Ostermann, Multi-grid dynamic iteration for parabolic equations, BIT, 27 (1987), pp. 216-234.

[21] M. S. MAHmoud, Robust Control and Filtering for Time-Delay Systems, CRC Press, 2000.

[22] S. VAndewalle AND M. J. Gander, Optimized overlapping Schwarz methods for parabolic PDEs with time-delay, Lect. Notes In Comput. Sci., 40 (2005), pp. 291-298.

[23] A. TALBOt, The accurate numerical inversion of Laplace transforms, J. Inst. Math. Appl., 23 (1979), pp. 97-120.

[24] L. N. TRefethen And J. A. C. Weideman, The exponentially convergent trapezoidal rule, SIAM Review, 56 (2014), pp. 385-458.

[25] S. Vandewalle, Parallel Multigrid Waveform Relaxation for Parabolic Problems, SpringerVerlag, 2013.

[26] S. Vandewalle and G. Horton, Fourier mode analysis of the multigrid waveform relaxation and time-parallel multigrid methods, Computing, 54 (1995), pp. 317-330.

[27] J. Wu, Theory and Applications of Partial Functional Differential Equations, Springer-Verlag, New York, 1996.

[28] S. L. Wu AND T. Z. HuANG, Quasi-optimized overlapping Schwarz waveform relaxation algorithm for PDEs with time-delay, Commun. Comput. Phys., 14 (2013), pp. 780-800.

[29] S. L. Wu, C. M. Huang And T. Z. Huang, Convergence analysis of the overlapping Schwarz waveform relaxation algorithm for reaction-diffusion equations with time delay, IMA J. Numer. Anal., 32 (2012), pp. 632-671.

[30] S. L. WU AND T. Z. HUANG, Schwarz waveform relaxation for a neutral functional partial differential equation model of lossless coupled transmission lines, SIAM J. Sci. Comput., 35 (2013), pp. A1161-A1191.

[31] S. H. XiAng, G. He And Y. J. Cho, On error bounds of Filon-Clenshaw-Curtis quadrature for highly oscillatory integrals, Adv. Comput. Math., 41 (2015), pp. 573-597.

[32] M. E. Yalçln, J. A. K. Suykens And J. P. L. VAndewalle, Cellular Neural Networks, Multi-Scroll Chaos and Synchronization, World scientific, 2005.

[33] B. Zubik-Kowal AND S. VANDEWAlLE, Waveform relaxation for functional differential equations, SIAM J. Sci. Comput., 21 (1999), pp. 207-226.

[34] T. ZHou, A stochastic collocation method for delay differential equations with random input, Adv. Appl. Math. Mech., 6 (2014), pp. 403-418.

[35] Y. ZHANG, Optimal error estimates of compact finite difference discretizations for the Schrödingerpoisson system, Commun. Comput. Phys., 13 (2013), pp. 1357-1388. 